\title{
Formin Activity and mDia1 Contribute to Maintain Axon Initial Segment Composition and Structure
}

\author{
Wei Zhang ${ }^{1,2}$ - María Ciorraga ${ }^{1}$ - Pablo Mendez ${ }^{1}$ - Diana Retana ${ }^{1}$ - Norah Boumedine-Guignon ${ }^{3} \cdot$ Beatriz Achón $^{1}$. \\ Michaël Russier $^{3} \cdot$ Dominique Debanne $^{3} \cdot$ Juan José Garrido $^{1,4}$ (D)
}

Received: 4 June 2021 / Accepted: 11 August 2021 / Published online: 30 August 2021

(C) The Author(s) 2021

\begin{abstract}
The axon initial segment (AIS) is essential for maintaining neuronal polarity, modulating protein transport into the axon, and action potential generation. These functions are supported by a distinctive actin and microtubule cytoskeleton that controls axonal trafficking and maintains a high density of voltage-gated ion channels linked by scaffold proteins to the AIS cytoskeleton. However, our knowledge of the mechanisms and proteins involved in AIS cytoskeleton regulation to maintain or modulate AIS structure is limited. In this context, formins play a significant role in the modulation of actin and microtubules. We show that pharmacological inhibition of formins modifies AIS actin and microtubule characteristics in cultured hippocampal neurons, reducing F-actin density and decreasing microtubule acetylation. Moreover, formin inhibition diminishes sodium channels, ankyrinG and $\beta I V$-spectrin AIS density, and AIS length, in cultured neurons and brain slices, accompanied by decreased neuronal excitability. We show that genetic downregulation of the mDial formin by interference RNAs also decreases AIS protein density and shortens AIS length. The ankyrinG decrease and AIS shortening observed in pharmacologically inhibited neurons and neuron-expressing mDial shRNAs were impaired by HDAC6 downregulation or EB1-GFP expression, known to increase microtubule acetylation or stability. However, actin stabilization only partially prevented AIS shortening without affecting AIS protein density loss. These results suggest that mDial maintain AIS composition and length contributing to the stability of AIS microtubules.
\end{abstract}

Keywords Axon initial segment $\cdot$ Formins $\cdot \mathrm{mDia} 1 \cdot$ Microtubules $\cdot$ AnkyrinG

\section{Introduction}

The axon initial segment (AIS) serves as a barrier that controls membrane protein diffusion and cytoplasmic transport towards the axon, maintaining neuronal polarity [1-5]. Simultaneously, the AIS is characterized by a high density

Juan José Garrido

jjgarrido@cajal.csic.es

Wei Zhang

zhangweitbas@pku.edu.cn

Instituto Cajal, CSIC, 28002 Madrid, Spain

2 Present address: College of Chemistry and Molecular Engineering, Peking University, Beijing, China

3 UNIS, INSERM, UMR 1072, Aix-Marseille Université, 13015 Marseille, France

4 Alzheimer's Disease and Other Degenerative Dementias, Centro de Investigación Biomédica en Red de Enfermedades Neurodegenerativas (CIBERNED), Madrid, Spain of voltage-gated ion channels that allow action potential (AP) generation [6]. This high density of voltage-gated ion channels is achieved by a not completely understood protein complex formed by scaffold proteins, such as ankyrinG or $\beta I V$-spectrin, and a specialized actin and microtubule cytoskeleton [7]. The whole complex is characterized by a high stability and detergent extraction resistance [8], but has a surprising high degree of plasticity [9], which regulatory mechanisms at cytoskeleton level are relatively unknown. AnkyrinG is the main scaffold protein that anchors voltage-gated sodium channels $[10,11]$ and is bound to actin cytoskeleton through $\beta I V-s p e c t r i n ~[12]$. Moreover, ankyrinG can also bind to microtubules through EB1/3 proteins [13].

AIS actin and microtubules contribute to control axonal traffic and AIS structure [3, 5, 14-18]. However, our understanding of the mechanisms controlling AIS cytoskeleton dynamics is limited. AIS microtubules are highly fasciculated and contain post-translational modifications of tubulin, such as acetylation and detyrosination, which allow kinesin-1 to transport cargoes into the axon [19-21]. Changes in tubulin 
acetylation by the tubulin deacetylase HDAC6 contribute to modifications in AIS [21, 22]. A higher phosphorylation of the microtubule-associated protein Tau, due to V337M mutation, contributes to AIS shortening [23]. Recently, TRIM46 (tripartite motif-containing protein 46 ) was identified as a microtubule fasciculation protein at the AIS [15], and the microtubule-associated protein MAP7D2 was also localized to the proximal axon promoting kinesin-1-mediated cargo transport [17]. Besides that, AIS actin cytoskeleton is necessary to maintain the cytoplasmic barrier that impairs somatodendritic cargoes to enter the axon [5, 16, 24]. Different actin structures have been described in the AIS, such as actin patches, actin rings, or actin filaments [25]. The AIS also contains an actin-rich structure, the cisternal organelle (CO), supposed to be a calcium regulatory compartment [26] and related to AIS plasticity [27]. Some AIS actin-related proteins have been identified, such as synaptopodin, $\alpha$-actinin- 2 , or myosin II and V [14, 24, 28]. Despite our increasing knowledge of actin- and microtubule-related proteins in the AIS, the mechanisms that regulate the crosstalk between both cytoskeleton components at the AIS are not fully understood.

Some studies have shown a mutual regulation of actin and microtubule stability in axons $[29,30]$. The crosstalk between actin and microtubules has been well-studied during acquisition of neuronal cell shape or cell migration, modulated by shared regulators, such as MAPs, EBs, or formins [29]. Among them, EB1/EB3 and MAP7D2 are localized at the AIS $[3,17]$, while only one previous study points to localization of formin 2 at the proximal axon [31]. Fifteen different formins have been described in mammals [32], defined by their most conserved formin homology 2 (FH2) domain [33], which is the core element for formin physiological functions [34]. The regulatory mechanisms of formins are not fully understood, but their activation and autoinhibition depend on different mechanisms $[35,36]$. Formins are a major class of effectors for cytoskeleton modulation, with the ability to modulate and coordinate actin and microtubule structures [34, 37, 38]. Among them, formins modulate actin filaments elongation and nucleation, and contribute to microtubule stabilization, promoting indirectly their acetylation [39]. Among their multiple physiological roles, formins are necessary to maintain apico-basal polarity in neuroepithelial and epithelial cells [40-42], and to regulate axonal actin structural dynamics [43]. Furthermore, formins are related to degenerative diseases and mental disorders [44].

Thus, we hypothesized that formins may play a role in the regulation of AIS structure. We found that formin inhibition modifies actin and microtubule AIS cytoskeleton and reduces scaffold and functional protein density at the AIS, both in cultured neurons and brain slices. This leads to a reduced intrinsic neuronal excitability. Moreover, formin activity has a dual role in microtubule or actin filament regulation leading to the maintenance of AIS length or AIS protein density. Finally, we have identified mDial formin as a main formin to maintain AIS protein density and AIS length.

\section{Materials and Methods}

\section{Animals}

Animals, Swiss CD1 mice, were housed in the Experimental Animal facility at Cajal Institute and maintained according to national legislation (53/2013, BOE no. 1337) and guidelines of the Council of the European Communities (2010/63/UE). Protocols were previously approved by the CSIC bioethics committee.

\section{Reagents and Plasmids}

The following reagents were used in the study: SMIFH2 (S4826), Tubastatin A hydrochloride (SML0044), and CK-666 (SML-0006) were purchased from Sigma-Aldrich, and jasplakinolide (2792) from TOCRIS. The interference RNA plasmids of mouse for mDial were obtained from Origene with the RFP tag. Two effective targets were identified: 5'-TGCCACTGACGAGAAGGACAAGTTTGTTG-3' (Cat\# TF500527D, shmDia1-1) and 5'-GAAG GAATCCTACTGCTGGTCAGAGCCAT-3' (Cat\# TF500527A, shmDia1-2). HDAC6 interference RNA plasmids with GFP tag from Origene have been previously described [21]. AnkG-GFP (270 kD) plasmid was a kind gift from Dr Van Bennett laboratory. pEGFP-N1, as a control of recombinant DNA over-expression, was bought from Clontech (Cat\# 6085-1). The GFP-CA-mDial vector expressing a constitutively active form of mDial (plasmid \# 45583) and the recombinant vector of EB1-GFP (plasmid \# 39299) were obtained from Addgene.

\section{Cell Cultures}

Mouse hippocampal neurons were prepared as previously described $[45,46]$. Briefly, mice hippocampi were dissected, incubated in a $0.25 \%$ trypsin solution in $\mathrm{Ca}^{2+} / \mathrm{Mg}^{2+}$ free Hank's buffered salt solution (HBSS) and dissociated using fire-polished Pasteur pipettes. Neurons were placed on poly-lysine-coated coverslips $(1 \mathrm{mg} / \mathrm{ml})$ at a density of 6000 cells $/ \mathrm{cm}^{2}$ for $2 \mathrm{~h}$ in a plating medium (MEM, $10 \%$ horse serum, $0.6 \%$ glucose and Glutamax-I). Then coverslips were inverted and transferred to culture dishes containing an astrocyte monolayer in a neuronal medium (Neurobasal, B27 supplement and Glutamax-I). A $5 \mu \mathrm{M} 1-\beta$-Darabinofuranosylcytosine (AraC) was added 2 days after to curb glial cells proliferation. Neurons were maintained replacing one-third of neuronal medium every week. In the case of 
pharmacological treatments in the absence of glial cell layer, coverslips were transferred to plates containing glial cellconditioned medium.

Nucleofection was performed after neuronal dissociation using the P3 Primary Cell 4D-Nucleofector ${ }^{\mathrm{TM}} \mathrm{X}$ Kit L for primary mammalian neural cells (Cat\# V4XP-3012, Lonza), using the CL133 program, $2 \times 10^{6}$ cells and $3 \mu \mathrm{g}$ of total DNA for each nucleofection. After nucleofection, neurons were plated at a density of $15.000 \mathrm{cells} / \mathrm{cm}^{2}$.

Neuronal transfection was performed after plating at the times described in the "Results" section. Hippocampal neurons $\left(20,000 \mathrm{cells} / \mathrm{cm}^{2}\right)$ were transfected with Lipofectamine 2000 (Cat\#11668-030, Invitrogen) and maintained for $24 \mathrm{~h}$ or $48 \mathrm{~h}$.

Neuro-2a cells were cultured in DMEM containing 10\% FBS and glutamine $(2 \mathrm{mM})$. Cells were transfected using Lipofectamine 2000 (Invitrogen) following manufacturer instructions, and kept for 3 days before fixation and immunocytochemistry.

\section{Brain Slice Cultures and Electrophysiology}

Slice cultures containing the hippocampus and entorhinal cortex were obtained from postnatal day 5-7 rats as previously reported [47]. Slices $(350 \mu \mathrm{m})$ were cut in ice-cold sucrosebased slicing solution (280 $\mathrm{mM}$ sucrose, $26 \mathrm{mM} \mathrm{NaHCO} 3$, $1.3 \mathrm{mM} \mathrm{KCl}, 1 \mathrm{mM} \mathrm{CaCl} 2,10 \mathrm{mM} \mathrm{MgCl} 2,11 \mathrm{mM}$ D-glucose, and $2 \mathrm{mM}$ kynurenate) oxygenated with 95\% O2/ $5 \% \mathrm{CO}_{2}$ and were collected in the slicing solution added with $5 \%$ horse serum and $0.6 \%$ HEPES $(1 \mathrm{M})$, and maintained for $1 \mathrm{~h}$ at room temperature in oxygenated $(95 \% \mathrm{O} 2 / 5 \% \mathrm{CO} 2)$ standard artificial cerebrospinal fluid $(125 \mathrm{mM} \mathrm{NaCl}, 2.5 \mathrm{mM}$ $\mathrm{KCl}$; $0.8 \mathrm{mM} \mathrm{NaH} 2 \mathrm{PO} 4,3 \mathrm{mM} \mathrm{NaHCO} 3,3 \mathrm{mM} \mathrm{CaCl}$, $2 \mathrm{mM} \mathrm{MgCl} 2$, and $11 \mathrm{mM}$ D-glucose). Each slice was placed on 320-mm latex PTFE hydrophilic membranes (Merck-MilliporeMillicell) inserted into 35-mm Petri dishes containing $1 \mathrm{ml}$ of culture medium $(25 \mathrm{ml}$ MEM, $13.5 \mathrm{ml}$ HBSS, $12.5 \mathrm{ml}$ horse serum, $0.5 \mathrm{ml}$ penicillin/streptomycin, $0.8 \mathrm{ml}$ glucose solution $(1 \mathrm{M}), 0.1 \mathrm{ml}$ ascorbic acid solution (1 $\mathrm{mg} / \mathrm{ml}), 0.4 \mathrm{ml} 4$-(2-hydroxyethyl)-1-piperazine ethanesulfonic acid (HEPES) $(1 \mathrm{M}), 0.5 \mathrm{ml} \mathrm{B} 27$, and $8.95 \mathrm{ml}$ water) and kept at $34{ }^{\circ} \mathrm{C}, 95 \% \mathrm{O} 2-5 \% \mathrm{CO}_{2}$. To arrest glial proliferation, $5 \mu \mathrm{M}$ Ara-C was added to the culture medium and removed the day after. Cultures at 7 DIV were treated in the culture medium for $3 \mathrm{~h}$ before recording with $30 \mu \mathrm{M}$ SMIFH2 diluted in DMSO or with DMSO alone (1\%).

Whole-cell patch-clamp recordings were obtained from CA3 pyramidal neurons. The external solution contained (mM): $125 \mathrm{NaCl}, 26 \mathrm{NaHCO} 3,3 \mathrm{CaCl} 2,2.5 \mathrm{KCl}, 2 \mathrm{MgCl} 2$, $0.8 \mathrm{NaH} 2 \mathrm{PO} 4$, and $10 \mathrm{D}$-glucose, and was equilibrated with $95 \% \mathrm{O} 2-5 \% \mathrm{CO}_{2}$. Patch-pipettes (5-10 $\mathrm{M} \Omega$ ) were filled with a solution containing $(\mathrm{mM}) 120$ potassium gluconate, $20 \mathrm{KCl}$, 0.5 EGTA, 10 HEPES, 2 Na2ATP, $0.3 \mathrm{NaGTP}$, and $2 \mathrm{MgCl}$, $\mathrm{pH}$ 7.4. Recordings were made at $30{ }^{\circ} \mathrm{C}$ in a temperature- controlled recording chamber (Luigs \& Neumann). The voltage and current signals were acquired on a $\mathrm{PC}$ computer fed by a Digidata1440A at a sampling frequency of $10 \mathrm{kHz}$ for input-output curves and $40 \mathrm{kHz}$ for spike threshold measurements. CA3 neurons were identified by their firing behavior. Neurons were held at their resting membrane potential $(\sim 77$ $\mathrm{mV})$. Input-output curves were established by $10 \mathrm{pA}$ increments of current step, and spikes were counted. Rheobase and gain were measured for each neuron as previously reported [48]. Spike threshold was measured as the first value of voltage corresponding to a rate exceeding $30 \mathrm{mV} / \mathrm{ms}$.

Recordings in P26 mice acute slices were from layer 2/3 neurons visually identified using infrared video microscopy. Slices were perfused with oxygenated artificial cerebrospinal fluid containing the following: $126 \mathrm{mM} \mathrm{NaCl}, 26 \mathrm{mM}$ $\mathrm{NaHCO}$, $2.5 \mathrm{mM} \mathrm{KCl}, 1.25 \mathrm{mM} \mathrm{NaH} 2 \mathrm{PO} 4,2 \mathrm{mM}$ $\mathrm{MgSO} 4,2 \mathrm{mM} \mathrm{CaCl}$, and $10 \mathrm{mM}$ glucose (pH 7.4). Recording were performed in the presence of kynurenic acid $(2 \mathrm{mM})$ and SR95531 (Gabazine, $10 \mu \mathrm{M})$ to block glutamate and GABAA receptor-mediated synaptic currents. Patchclamp electrodes contained intracellular solution composed of $70 \mathrm{mM} \mathrm{K}^{+}$-gluconate, $70 \mathrm{mM} \mathrm{KCl}, 2 \mathrm{mM} \mathrm{NaCl}, 2 \mathrm{mM}$ $\mathrm{MgCl} 2,10 \mathrm{mM}$ HEPES, $1 \mathrm{mM}$ EGTA, $2 \mathrm{mM} \mathrm{MgATP}$, and $0.3 \mathrm{mM} \mathrm{Na} 2 \mathrm{GTP}$ (pH 7.3). A 3-s ramp between 0 and $250 \mathrm{pA}$ was used to calculate threshold for action potential generation.

\section{Immunocytochemistry}

Neurons or Neuro2a cells were fixed in $4 \%$ paraformaldehyde (PFA) for $15 \mathrm{~min}$, washed in phosphate buffered saline (PBS), treated with $50 \mathrm{mM} \mathrm{NH}_{4} \mathrm{Cl}$ for $10 \mathrm{~min}$, and incubated at RT in blocking buffer for $1 \mathrm{~h}(0.22 \%$ gelatin, $0.1 \%$ Triton X-100 in PBS). Primary antibodies were incubated for $1 \mathrm{~h}$ diluted in blocking buffer. The primary antibodies used were rabbit polyclonal anti-DIAPH1 (1:70, Cat\# PA5-27607, Thermo Fisher Scientific), knockout-validated rabbit polyclonal anti-DAPH1 (1:100, ab129167, Abcam), mouse monoclonal anti-mDia1 (1:50, 610849, BD, previously used in [49]), mouse monoclonal anti-AnkG (IgG2a, 1:150, clone N106/36, NeuroMab), mouse monoclonal anti-PanNaCh (IgG1, 1:100, Cat\# S8809, Sigma-Aldrich), rabbit polyclonal anti-pMLC (1:200, Cat\# PA5-17727, Thermo Fisher Scientific), rabbit polyclonal anti- $\beta$ IV-Spectrin (1:1000, a gift of Dr Matthew Rasband, Baylor College of Medicine), chicken polyclonal anti-MAP2 (1:5000, Cat\# ab5392, Abcam), mouse monoclonal anti-acetylated-tubulin (IgG2b, 1:5000, Cat\# T7451, Sigma-Aldrich), mouse monoclonal anti- $\alpha$-tubulin (IgG1, 1:5000, Cat\# T6199, Sigma-Aldrich), rabbit polyclonal anti-KIF5C (1:200, Cat\# ab5630, Abcam), and rabbit polyclonal anti-Synaptopodin (1:500, Cat\# S9442, Sigma-Aldrich). Coverslips were incubated with Alexa coupled isoform specific secondary antibodies (1:1000) in blocking buffer for $45 \mathrm{~min}$. F-Actin was stained using Alexa Fluor-568-conjugated 
Phalloidin (1:100, Cat\# A12380, Thermo Fisher Scientific). Coverslips were mounted with Fluoromount-G (Southern-Biotech). Images were acquired on a Leica SP5 confocal microscope at a resolution of $1024 \times 1024$ pixels.

\section{Immunohistochemistry}

Brains from mice P26-P30 days old were quickly removed and acute coronal slices $(300 \mu \mathrm{m})$ containing sensorimotor cortex were cut with a vibratome $\left(4^{\circ} \mathrm{C}\right)$ in a solution containing: $234 \mathrm{mM}$ sucrose, $11 \mathrm{mM}$ glucose, $26 \mathrm{mM} \mathrm{NaHCO}_{3}$, $2.5 \mathrm{mM} \mathrm{KCl}, 1.25 \mathrm{mM} \mathrm{NaH}_{2} \mathrm{PO}_{4}, 10 \mathrm{mM} \mathrm{MgSO}_{4}$, and $0.5 \mathrm{mM} \mathrm{CaCl}_{2}$ (equilibrated with $95 \% \mathrm{O}_{2}-5 \% \mathrm{CO}_{2}$ ). SMIFH2 treatment was carried out at a concentration of 15 or $30 \mu \mathrm{M}$ for $3 \mathrm{~h}$, and the same volume of vehicle DMSO was used as control. Slices were fixed $30 \mathrm{~min}$ at RT with $4 \%$ PFA and washed in PBS. Slices were incubated for $2 \mathrm{~h}$ at RT with PBS containing $0.1 \%$ TritonX-100 and $10 \%$ goat serum, followed by $4{ }^{\circ} \mathrm{C}$ overnight incubation with anti-AnkG antibody (IgG2a, 1:150) in PBS containing 0.1\% TritonX-100 and $1 \%$ goat serum. After secondary antibody incubation (1:500) for $2 \mathrm{~h}$ at RT, sections were counterstained with bis-benzimide ( $5 \mu \mathrm{g} / \mathrm{ml}$, Cat\# B2261, Sigma-Aldrich) for nuclei staining and mounted in Fluoromount-G. Images were acquired on a Leica SP5 confocal microscope using Leica DM6000B 40 $\times 1.25$ N.A. oil objective. Sections were imaged in $\mathrm{z}$ stacks with a $0.5-\mu \mathrm{m}$ step size. For qualitative analysis, a Z-projection was obtained in the Fiji-ImageJ software. We measured individual AISs in cortical layers II/III, IV, and V/VI. Cortical layers were distinguished according to nuclei patterns. In the case of CA1 staining quantification, total ankyrinG fluorescence was measured in the whole CA1 region as shown in figures.

\section{Fluorescence Recovery After Photobleaching}

For FRAP experiments, hippocampal neurons were plated directly on $35-\mathrm{mm}$ culture dishes with glass bottom (Ibidi). Neurons were plated at a density of 20.000 cells $/ \mathrm{cm}^{2}$ and supporting glial cells were place on inverted 24-mm coverslips. Neurons were lipofected with AnkyrinG-GFP at 8 DIV and kept for $24 \mathrm{~h}$. Prior to FRAP experiment, neurons were treated with $15 \mu \mathrm{M}$ SMIFH2 or DMSO for $3 \mathrm{~h}$. Live imaging was performed on a Leica SP5 inverted confocal microscope, using $63 \times 1.40 \mathrm{NA}$ oil objective and maintaining $37^{\circ}$ $\mathrm{C}$ and $5 \% \mathrm{CO}_{2}$ in the live cell chamber. Time-lapse images were acquired using bidirectional scan at $1400 \mathrm{~Hz}$ with the format of $256 \times 256$ pixels and the pinhole size was 2 airy units. AIS was distinguished by AnkyrinG-GFP concentration at the proximal axon. A ROI with an area of $5 \mu \mathrm{m} \times 5 \mu \mathrm{m}$ on the proximal axon was photobleached at $100 \%$ 488-nm Laser Line power with $25 \%$ Argon Laser Power, reducing local fluorescence by $80-95 \%$. FRAP was performed with 10 frames of $0.1-\mathrm{s}$ intervals for pre-bleach and $15 \mathrm{~s}$ for bleach with 0.1 -s intervals, followed by post-bleach with 240 frames of 0.5 -s intervals. FRAP analysis was performed as previously described [13]. The recovery curve was generated by pooling together at least 15 neurons in each condition and averaging each frame the recovery rate. The maximum recovery percentage was obtained through averaging recovery data in $20 \mathrm{~s}$ at the plateau from every neuron in Sigmaplot v12.5 (Systat Software Inc., San Jose, CA, USA).

\section{AIS Parameter Quantification}

Confocal microscope settings were adjusted to prevent signal saturation and the images were taken in $\mathrm{z}$ stacks with a $0.5-\mu \mathrm{m}$ step size. Images were obtained randomly. All images from every experiment were obtained using the same settings, standardized in the control samples of each experiment. For quantitative analysis, all coverslips in each experiment experienced the same procedures for labeling. AIS was identified by ankyrinG staining. A stack of $1.5-\mu \mathrm{m} 3$ confocal sections containing the AIS was used to quantify AIS parameters. Analysis was performed using the Fiji-ImageJ software (NIH) drawing a line along the axon, starting at the soma. Data were smoothed every $1 \mu \mathrm{m}$ using the Sigma Plot 12.5 software. AIS start, maximum, and end positions were determined as described previously [50]. Total fluorescence intensity for each marker was obtained by adding fluorescence intensity values from start to end position after determining AIS length using ankyrinG staining. Data where normalized in each experiment to the value of the mean fluorescence in control neurons. For AIS profiles data were normalized to the maximum fluorescence value in control neurons.

\section{Statistical Analysis}

All statistical analyses were carried out in GraphPad Prism 5 and Sigmaplot v12.5. Sample Gaussian distributions were first assessed for normality using the D'Agostino and Pearson omnibus test or Shapiro-Wilk's test. Statistical analysis was performed using a two tailed t-test for two group comparisons: Unpaired $t$-test for parametric data and a post hoc Mann-Whitney test for non-parametric data. For multiple group comparisons, one-way analysis of variance with Tukey post hoc test was used for parametric data and a post hoc Kruskal-Wallis with Dunn's test for non-parametric data. Graphs were represented as the mean \pm SEM. Differences were considered significant when $p$-value less than 0.05 , and represented as $* p<0.05, * * p<0.01, * * *<0.001$. 


\section{Results}

\section{Formin Inhibition Modify AIS Protein Density}

To determine the effects of formin activity on AIS structure and protein composition, we treated neurons with SMIFH2, an inhibitor of formin conserved FH2 domain [34, 37, 51]. Treatment with SMIFH2 $(15 \mu \mathrm{M})$ in 14 DIV-cultured hippocampal neurons led to a significant ankyrinG decrease after $3 \mathrm{~h}$ of application $(-25 \%$, Fig. 1A, B) that was detected after $30 \mathrm{~min}(-15 \%$, Fig. 1C). This decrease was more pronounced in young neurons (7 DIV,$-32 \%)$, than in older neurons (21 DIV,$-15 \%$ ) after $3 \mathrm{~h}$ of application (Fig. 1B), and was due to a decrease all along the AIS (Fig. 1D). Moreover, a similar reduction was also detected in 14 DIV neurons for other AIS proteins (Fig. 1E), such as $\beta \mathrm{IV}$-spectrin $(-20 \%)$, voltage-gated sodium channels ( $\mathrm{PanNaCh},-20 \%)$, and phospho-myosin light chain (pMLC, $-10 \%$ ), suggesting an effect on the underlying AIS cytoskeleton (Fig. S1). To discard that SMIFH2 treatment was generating an indirect effect on neurons through formin inhibition in astrocytes, we applied SMIFH2 to neurons in the absence of astrocytes (Fig. $\mathrm{S} 2 \mathrm{~A})$ and detected again an ankyrinG reduction $(-28.80 \pm$ $1.89 \%$ ) compared to DMSO-treated neurons. Moreover, after additional incubation in new plates containing astrocyte-conditioned neuronal medium for $6 \mathrm{~h}$ (Fig. S2B), a further decrease of $10 \%$ of ankyrinG intensity was observed. After $24 \mathrm{~h}$, ankyrinG decrease showed an inverse correlation with increased expression of the somatodendritic marker MAP2 along the AIS, entering the axon (Fig. S2C-E). Finally, we analyzed whether ankyrinG decrease could be due to calpain activation as previously described $[45,52]$. Hippocampal neurons (14 DIV) were pre-treated with the calpain inhibitor MDL-28170 (50 nM) for $1 \mathrm{~h}$, and then neurons were incubated with SMIFH2 $(15 \mu \mathrm{M})$ or DMSO for $3 \mathrm{~h}$ (Fig. S3). Our results show that ankyrinG decrease is not impaired by calpain inhibition, and other mechanisms are responsible for AIS protein reduction.
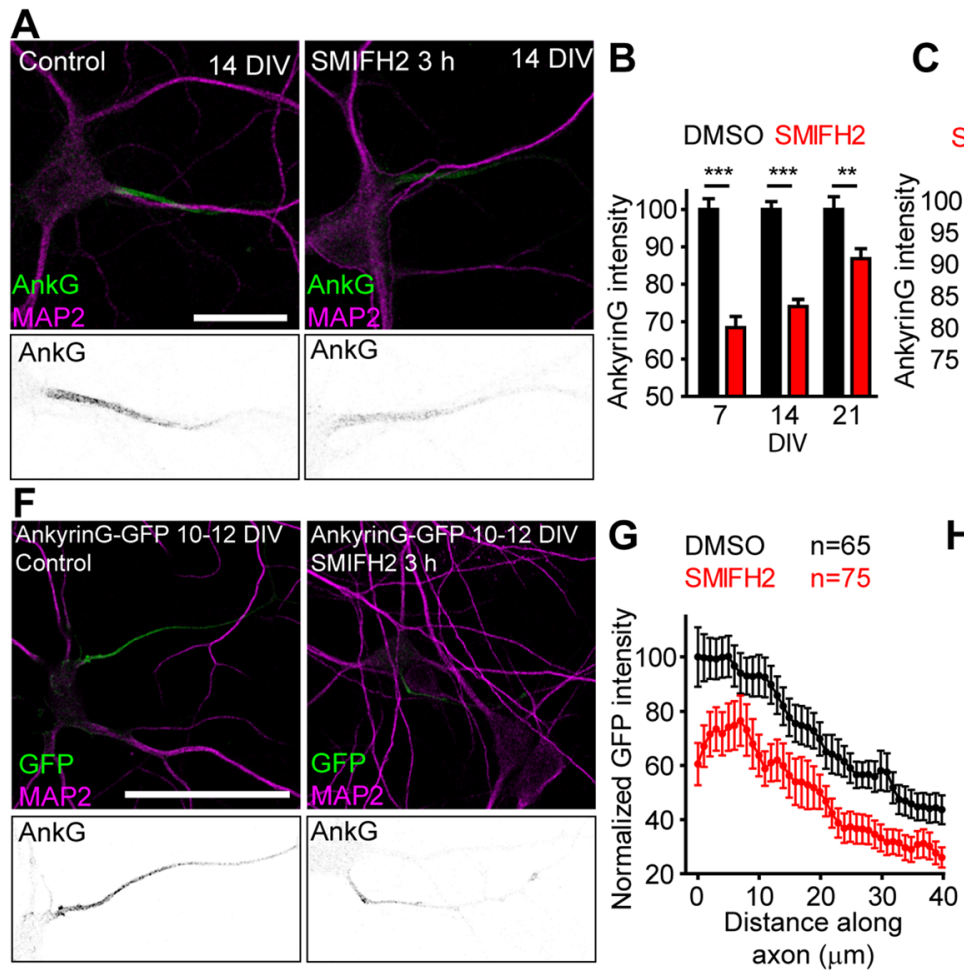

C

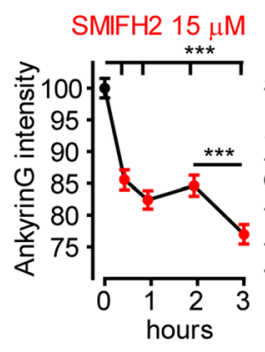

H

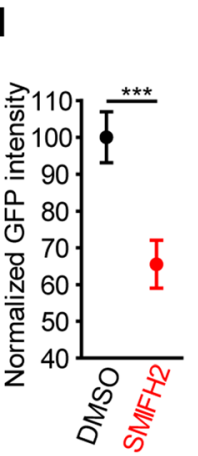

D

14 DIV

DMSO SMIFH2

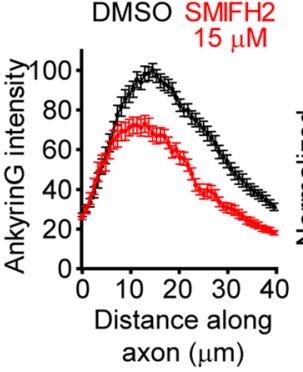

E

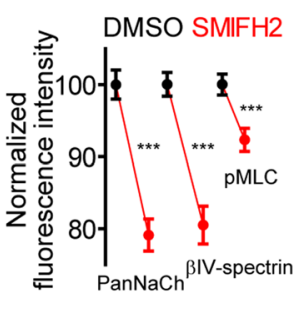

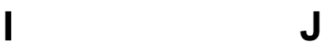
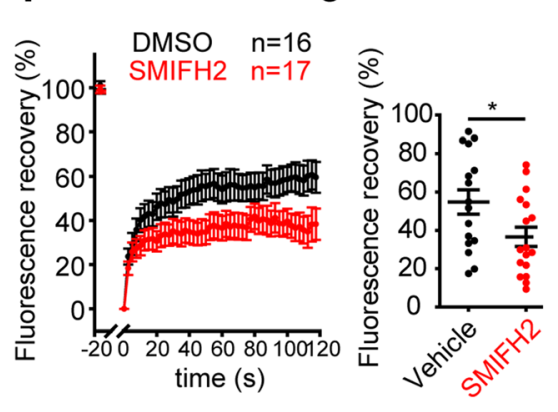

Fig. 1 Formin inhibition decreases AIS protein density in hippocampal neurons. A A total of 14 DIV hippocampal neurons treated for $3 \mathrm{~h}$ with DMSO or $15 \mu \mathrm{M}$ SMIFH2. AISs and somatodendritic compartment are identified by ankyrinG (green) and MAP2 (magenta). Bottom panels show AIS magnifications. Scale bar $=20 \mu \mathrm{m}$. B Normalized ankyrinG intensity in 7, 14, and 21 DIV hippocampal neurons treated for $3 \mathrm{~h}$ with vehicle (black bars) or $15 \mu \mathrm{M}$ SMIFH2 (red bars). **p $<0.01$, *** $p<$ 0.0001 , Mann-Whitney test ( $n=150$ /experimental condition). C Normalized ankyrinG fluorescence intensity in 14 DIV neurons treated with DMSO (black dots) or $15 \mu \mathrm{M}$ SMIFH2 (red dots) for $0.5,1,2$, and 3 h. *** $p<0.001$, Kruskal-Wallis, Dunn's multiple comparison test ( $n=$ 150/experimental condition). D AnkyrinG intensity profile in 14 DIV neurons along the AIS. E Normalized AIS sodium channels
(PanNaCh), $\beta I V-s p e c t r i n$, and phospho-myosin light chain (pMLC) fluorescence intensity in 14 DIV neurons treated with DMSO (black) or $15 \mathrm{mM} \mathrm{SMIFH} 2$ (red) for 3 h. $* * * p<0.001$, unpaired $t$-test $(n=120)$. F 10 DIV hippocampal neurons transfected with AnkyrinG-GFP for $48 \mathrm{~h}$ and exposed to DMSO or $15 \mu \mathrm{M}$ SMIFH2 for $3 \mathrm{~h}$. Bottom panels show AIS magnifications. Scale bar $=50 \mu \mathrm{m}$. G, H Normalized AIS GFP fluorescence intensity in vehicle (black) or SMIFH2 (red)-treated neurons $(\mathbf{H})$ and GFP signal profile along the AIS $(\mathbf{G}) . * * * p<0.001$, unpaired $t$ test. I Percentage of AIS AnkyrinG-GFP fluorescence recovery for $120 \mathrm{~s}$ after photobleaching in FRAP experiments. $\mathbf{J}$ Mean maximal GFP recovery. ${ }^{*} p<0.05$, unpaired $t$ test. Graphs show the mean \pm SEM of the indicated number of data obtained from at least three independent experiments. 
In order to confirm these results, 10 DIV hippocampal neurons were transfected with a plasmid expressing ankyrinGGFP for $48 \mathrm{~h}$ and treated with SMIFH2 for $3 \mathrm{~h}$ (Fig. 1F). Analysis of the GFP signal at the AIS confirmed the results obtained for endogenous ankyrinG. The ankyrinG-GFP signal was reduced by a $35 \%$ along the AIS (Fig. 1G, H). In addition, ankyrinG-GFP signal was analyzed "in vivo" in hippocampal neurons after SMIFH2 treatment (Fig. 1I, J). Fluorescence recovery after photobleaching (FRAP) experiments demonstrated that ankyrinG-GFP signal recovery after photobleaching was significantly lower after SMIFH2 treatment ( $36 \pm 5 \%$ vs $55 \pm 6 \%$ in control neurons, Fig. $1 \mathrm{~J}$ ).

We next analyzed whether this ankyrinG decrease by formin inhibition also happens in P26 mice brain slices (300 $\mu \mathrm{m})$ after 3-h incubation with vehicle (DMSO) or SMIFH2 $(15$ and $30 \mu \mathrm{M})$. We measured ankyrinG fluorescence intensity in hippocampus (Fig. 2A) and cortical layers II/III, IV, or V/VI (Fig. 2D). AnkyrinG intensity was reduced around $20 \%$ after $30 \mu \mathrm{M}$ SMIFH 2 treatment $(79 \pm 5 \%$ vs $100 \pm 3 \%$ in control slices) in CA1 hippocampal areas (Fig. 2B). SMIFH2 $(15 \mu \mathrm{M}$ or $30 \mu \mathrm{M})$ decreased ankyrinG intensity in an analogous percentage ( $-20 \%$ or $-30 \%$, respectively) in individual AISs in all cortical layers (Fig. 2C, D), showing a SMIFH2 concentration-dependent ankyrinG reduction (Fig. $2 \mathrm{C}$ ). These data suggest that formin activity is necessary to maintain AIS protein density, both in cultured neurons and in brain slices.

\section{Formin Inhibition Decreases Neuronal Intrinsic Excitability}

Since formin inhibition affects AIS structure, we analyzed whether action potential generation and neuronal excitability was altered. We treated organotypic slice cultures with SMIFH2 or DMSO for $3 \mathrm{~h}$ and recorded in CA3 pyramidal neurons. SMIFH2 treatment produced a marked reduction in intrinsic excitability (Fig. 2E). This reduction in excitability was associated with a higher rheobase (from $79 \pm 9 \mathrm{pA}, n=9$ to $133 \pm 10 \mathrm{pA}, n=9$, Mann-Whitney, $p<0.01$; Fig. $2 \mathrm{H}$ ), and a reduction in the gain (from $0.14 \pm 0.02$ to $0.07 \pm 0.01$ spike/ pA, Mann-Whitney, $p<0.01$; Fig. 2I). However, membrane capacitance and input resistance were found to be unchanged (DMSO: $293 \pm 5 \mathrm{pF}$ and $279 \pm 7 \mathrm{M} \Omega, n=9$, SMIFH2: $313 \pm 8$ $\mathrm{pF}$ and $226 \pm 5 \mathrm{M} \Omega$, Mann-Whitney $p>0.1$ and $p>0.05$ respectively). Furthermore, the voltage threshold of the action potential was found to be depolarized after treatment with SMIFH2 (DMSO: $-51 \pm 1 \mathrm{mV}, n=9$, SMIFH2: $-46.9 \pm$ $1.1 \mathrm{mV}, n=9$; Mann-Whitney, $p=0.01$; Fig. 2F, G). A similar depolarization of the spike threshold was found in P26 cortical neurons in acute brain slices (DMSO: $-39.04 \pm 1.06 \mathrm{mV}, n=$ 12, SMIFH2: $-36.08 \pm 0.71 \mathrm{mV}, n=13$; Mann-Whitney, $p=$ $0.009)$. No significant change was observed in spike amplitude in hippocampal neurons (DMSO: $69.5 \pm 3.2 \mathrm{mV}, n=9 \mathrm{vs}$
SMIFH2: $66.6 \pm 2.4 \mathrm{mV}, n=9$, Mann-Whitney $U$-test, $p=$ $0.66)$. These results suggest that formin inhibition has an impact on action potential generation due to ankyrinG and sodium channel reduction at the AIS.

\section{Formin Inhibition Decreases AIS F-Actin and Microtubule Acetylation}

Since formins remodel the cytoskeleton [38, 39], we analyzed actin and microtubule properties at the AIS after SMIFH2 treatment. First, we analyzed F-actin at the AIS in 14 DIV neurons treated for $3 \mathrm{~h}$ with DMSO or SMIFH2 $(15 \mu \mathrm{M})$ (Fig. 3A). F-Actin was identified using phalloidin, and we observed a previously described actin patches staining at the AIS [16]. F-actin intensity was measured along the ankyrinG staining in confocal sections of control or SMIFH2-treated neurons ( $n=165$, Fig. 3B, C). Phalloidin staining showed a $20 \%$ reduction of F-actin all along the AIS (Fig. 3B) in SMIFH2-treated neurons ( $82 \pm 2 \%$ vs $100 \pm 2.26 \%$ in control neurons, Fig. 3C). Quantification of ankyrinG in the same neurons showed a 25\% reduction in SMIFH2-treated neurons along the AIS (Fig. 3D). In contrast, ankyrinG intensity was not modified by inhibition of other important actin regulator, Arp2/3, using CK-666 (50 $\mu \mathrm{M})$ for $3 \mathrm{~h}$ (Fig. 3E). Then, we analyzed the actin-related cisternal organelle at the AIS (Fig. 3F), using its characteristic synaptopodin staining [53]. Only $25 \pm 5 \%$ of neurons treated with SMIFH2 were synaptopodin-positive at the AIS, compared to $90 \pm 2 \%$ of DMSO-treated neurons (Fig. 3G). Actin stabilization by jasplakinolide $(10 \mathrm{nM})$ pretreatment for $1 \mathrm{~h}$ increased the percentage of neurons with a detectable cisternal organelle $(59 \pm 5 \%)$ after SMIFH2 treatment (Fig. $3 \mathrm{H})$. However, jasplakinolide did not prevent ankyrinG intensity reduction (Fig. 3E), suggesting that ankyrinG reduction due to formin inhibition involves an actin-independent mechanism.

Thus, we analyzed whether formin inhibition was modifying microtubule characteristics leading to AIS protein density reduction. Actually, formins also contribute to increase microtubule acetylation and stability [39, 54]. Therefore, we quantified immunofluorescence intensity of acetylated- $\alpha$-tubulin after detergent extraction in control and SMIFH2-treated neurons (Fig. 4A). SMIFH2-treated neurons showed a significant decrease in acetylated- $\alpha$-tubulin staining $(46 \pm 8 \%)$ compared to $100 \pm 4 \%$ in control neurons (Fig. 4B). AnkyrinG and $\beta I V$-spectrin signals were also significantly decreased (71 $\pm 4 \%$ and $56 \pm 3 \%$ of the control level, respectively). Moreover, analysis of acetylated- $\alpha$-tubulin vs total tubulin ratio without detergent extraction shows a significant reduction in the AIS $(0.82 \pm 0.02$ vs $1.0 \pm 0.02$ in DMSO neurons) that was not found in proximal dendrites (Fig. 4C). Kinesin-1 selectively interacts with 

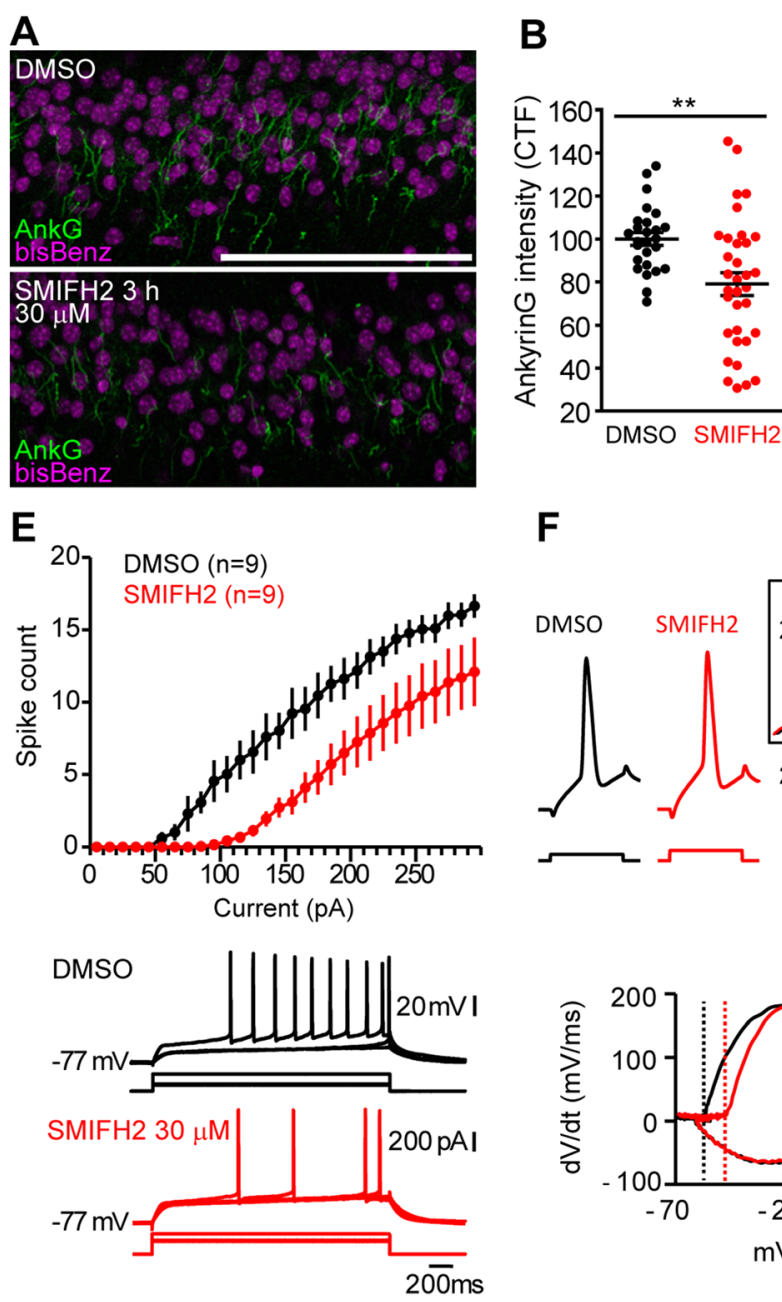

C

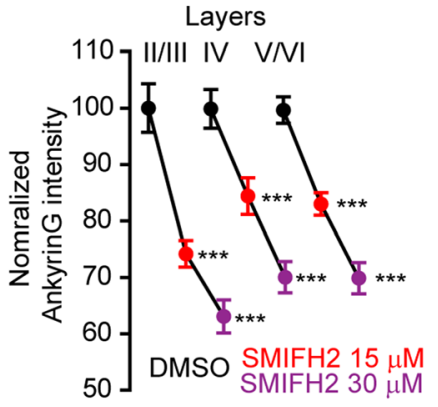

$\mathbf{F}$
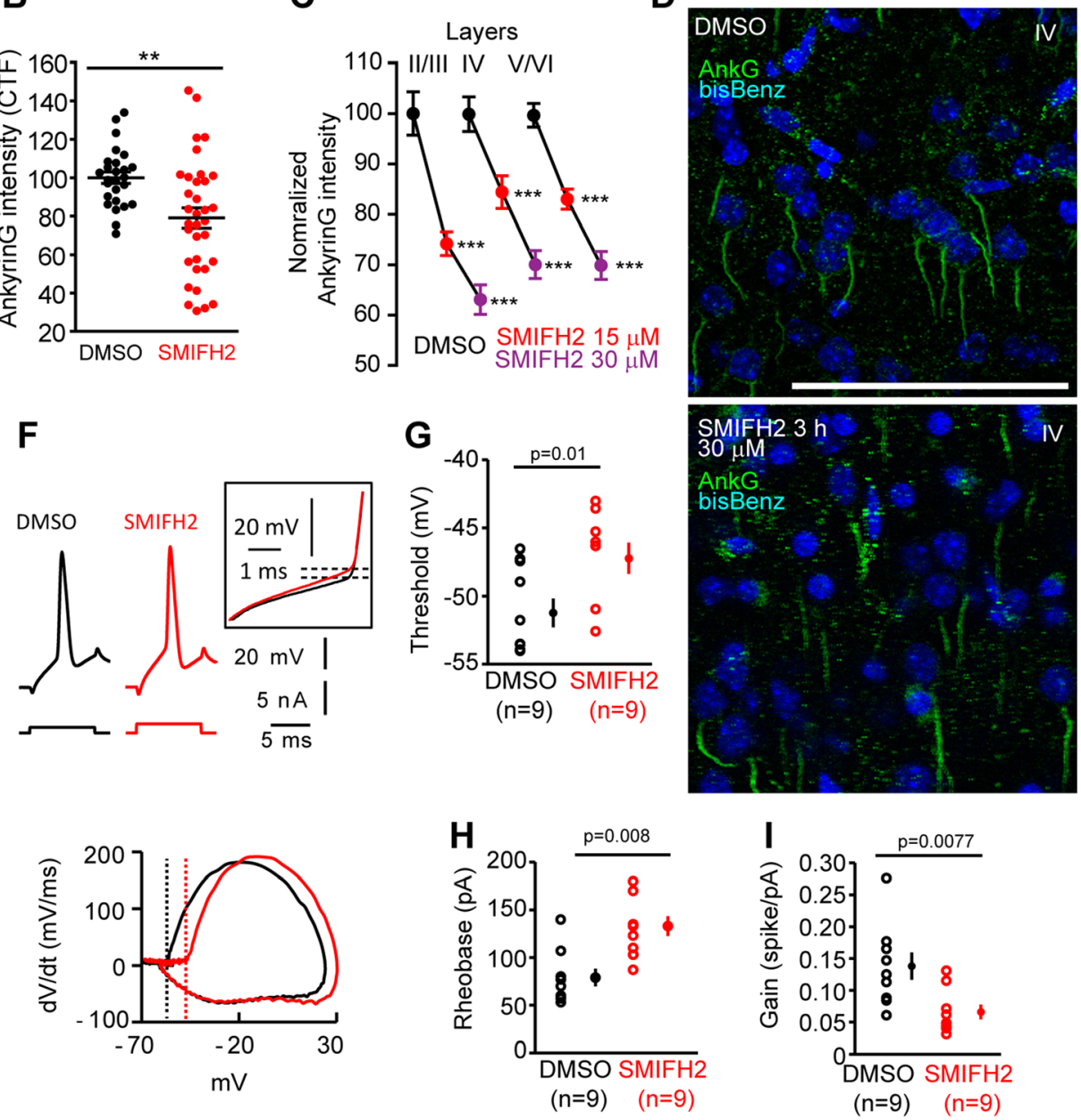

Fig. 2 Formin inhibition decreases ankyrinG intensity in brain slices and reduces neuronal intrinsic excitability. A Brain slices $(300 \mu \mathrm{m})$ containing hippocampi from P26 mice were treated with DMSO or $30 \mu \mathrm{M}$ SMIFH2 for $3 \mathrm{~h}$. Brain slices were stained to visualize ankyrinG (green) and nuclei (magenta). Scale bar $=100 \mu \mathrm{m}$. B Quantification of corrected total fluorescence intensity (CTF) in CA1 regions. ${ }^{*} p<0.01$, unpaired $t$ test. Each dot represents a CA1 zone as shown in A. Analysis was done in 4 independent experiments using 8 animals, and a total of 26 (control) and 34 (SMIFH2) slices. C Normalized ankyrinG intensity in DMSO (black) or $15 \mu \mathrm{M}$ (red) and $30 \mu \mathrm{M}$ SMIFH2 (magenta) in AISs of layers II/III, layer IV, and layers V/VI of P26 mice coronal sensorimotor cortex slices $(300 \mu \mathrm{m})$. $* * * p<$

acetylated microtubules guiding this motor into the axon $[19,55]$. Thus, we analyzed KIF5C distribution after SMIFH2 treatment. KIF5C showed a significant increase in 5 DIV neurons soma $(124 \pm 4.62 \%)$ accompanied by a reduction in axons $(89 \pm 4 \%)$ compared to DMSO-treated neurons (Fig. 4D, E). We detected a $30 \%$ KIF5C reduction in the first 30 microns of the axon compared to that in control cells (Fig. 4F). The above results suggest a participation of formin activity on AIS protein maintenance through a mechanism that involves microtubules.
0.001, Kruskal-Wallis, Dunn's multiple comparison test were analyzed. Data are represented as the mean \pm SEM and acquired in 150 AISs, for each condition and layer, from three independent experiments. D Representative images of ankyrinG-stained cortical sections treated with DMSO or SMIFH2. E CA3 neurons from rat hippocampal slice organotypic cultures treated for $3 \mathrm{~h}$ in the presence of $30 \mu \mathrm{M}$ SMIFH2 (red) or DMSO alone (control, black) were recorded under current-clamp and the number of evoked action potentials plotted against injected current. Representative traces are shown in the bottom panel. F, G Spike threshold, $\mathrm{dV} / \mathrm{dt}$ curves, and representative traces (inset $=$ focus on spike threshold) in control and SMIFH2-treated slices. $\mathbf{H}$, I Rheobase (H) and gain (I) in control (black) and SMIFH2 (red)-treated slices

\section{mDia1 Contributes to Maintain AIS Proteins}

A previous study in neurons confirmed that mDial is the primary target of SMIFH2 activity on microtubules in hippocampal neurons [49]. Moreover, mDial is necessary to maintain neuroepithelial cell polarity [42] and modulates axon and dendrites structures $[49,56]$, being related to microtubule stability and acetylation, and actin dynamics modulation [54]. Thus, we stained 14 DIV hippocampal neurons with 3 different mDial antibodies (see "Methods" section), one of them validated in mDial knockout cells (Abcam). The staining 


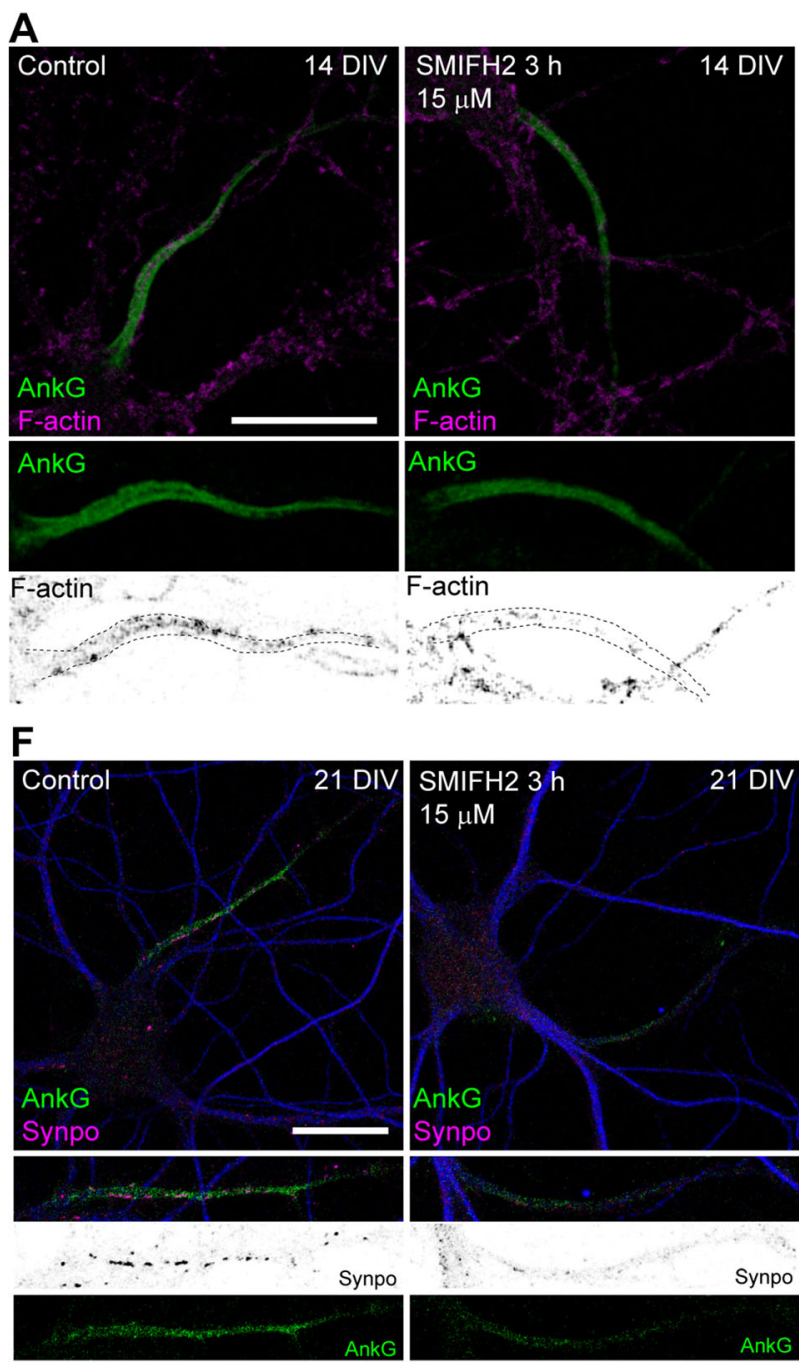

Fig. 3 Formin inhibition decreases F-actin intensity in the AIS and affects cisternal organelle. A A total of 14 DIV neurons treated with DMSO or $15 \mu \mathrm{M}$ SMIFH 2 for $3 \mathrm{~h}$. AnkyrinG staining (green) identifies the AIS and Alexa Fluor 568 Phalloidin (magenta) stains F-actin. Magnifications of AISs are shown in bottom panels. Scale bar $=20 \mu \mathrm{m}$. B, C Normalized Factin intensity along the AIS (B) and normalized total F-actin within the AIS (C) in vehicle (black) or SMIFH2 treated neurons (red). ***p $<$ 0.0001 , Mann-Whitney test. D Normalized ankyrinG profile and total ankyrinG intensity within the AIS in the same neurons quantified in $\mathbf{B}$ and C. E Normalized ankyrinG fluorescence intensity in 14 DIV hippocampal neurons treated with the Arp2/3 inhibitor CK-666 $(50 \mu \mathrm{M})$. F AnkyrinG (green) and cisternal organelle (synaptopodin, magenta) staining in 21 DIV hippocampal neurons treated with vehicle DMSO or
B
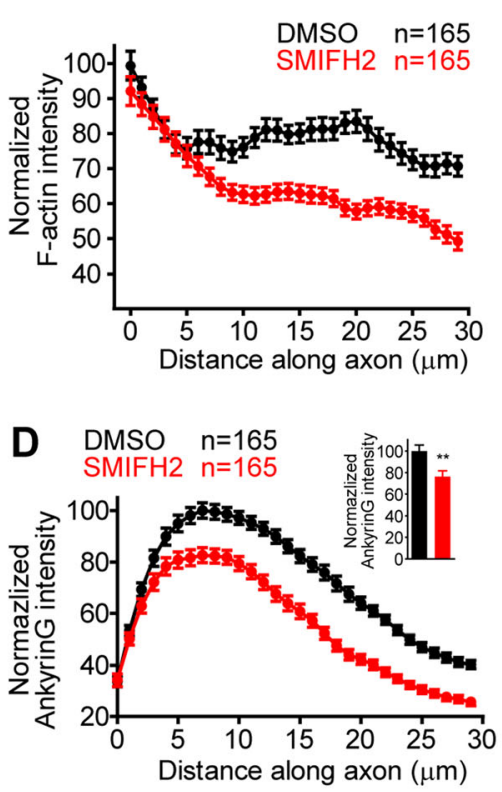

$\mathbf{E}$

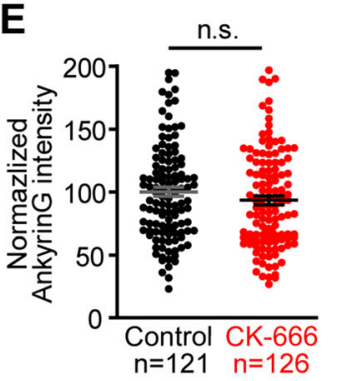

G
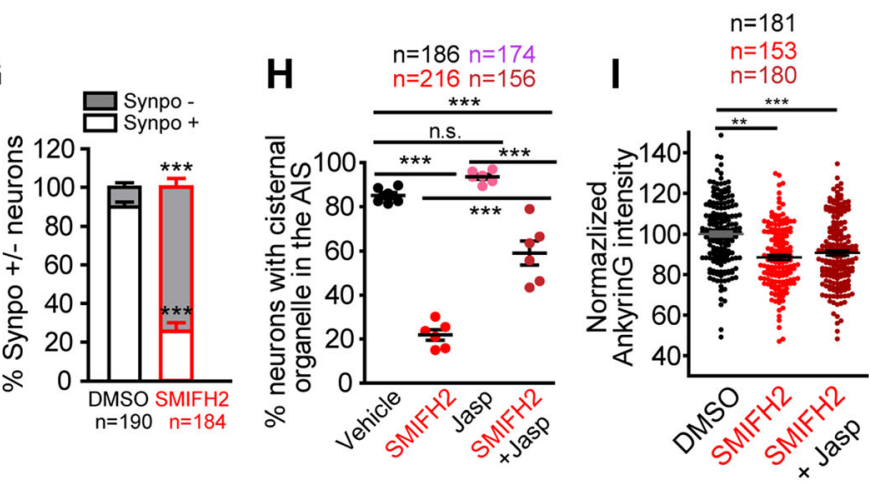

$15 \mu \mathrm{M}$ SMIFH2 for $3 \mathrm{~h}$. MAP2 staining (blue) identifies dendrites. Magnifications of AIS region are shown in bottom panels. Scale bar $=$ $20 \mu \mathrm{m}$. G Percentage of neurons with or without synaptopodin puncta in the AIS in the presence or absence of SMIFH2. $* * p<0.01$, MannWhitney test. H Percentage of synaptopodin puncta-positive neurons in the AIS of 21 DIV hippocampal neurons treated with $15 \mu \mathrm{M}$ SMIFH 2 in the presence or absence of $10 \mathrm{nM}$ jasplakinolide for $3 \mathrm{~h}$. $* * * p<0.01$, Mann-Whitney test. Data were acquired from 6 independent experiments and represented as the mean \pm SEM. I Normalized ankyrinG intensity in 14 DIV hippocampal neurons treated for $3 \mathrm{~h}$ with $15 \mu \mathrm{M}$ SMIFH2 alone or in combination with $10 \mathrm{nM}$ Jasplakinolide $* * p<0.01$, *** $p<0.001$, Kruskal-Wallis, Dunn's multiple comparison test. Data were acquired from 3 independent experiments and represented as the mean \pm SEM pattern of all three mDia1 antibodies was similar and not specifically enriched at the AIS, though mDial staining appeared at the AIS (Fig. S4A-C). All three antibodies stained mainly the soma with a Golgi and perinuclear staining, as well as dendrites and axons (Fig. S4A-C). We observed a similar pattern in hippocampal neurons expressing CAmDia1-GFP (Fig. S4D). To analyze the potential role of mDial on AIS modulation, we used two different mDial interference RNAs (shmDia1-1, shmDia1-2) that express RFP protein as reporter
(Fig. S5). Interference RNAs were validated in Neuro2a cells by immunofluorescence using the mDial knockout-validated antibody (Abcam). Both interference RNAs decreased mDia1 signal by $70 \%, 3$ days after transfection, compared to cells transfected with scrambled shRNA (Fig. S5A-D).

To analyze mDial shRNA effects on neurons, first we checked that mDial shRNA was not affecting axon and dendrite development and did not find differences between scrambled shRNA- and shmDia1-1-nucleofected neurons regarding 
Fig. 4 Formin inhibition modifies microtubules in the AIS and axonal transport. A Images of acetylated-tubulin (green), ankyrinG (red) and $\beta I V$-spectrin (blue) in the AIS of 13 DIV control and SMIFH2-treated neurons after detergent extraction for $5 \mathrm{~min}$ before fixation. Scale bar $=$ $10 \mu \mathrm{m}$. B Normalized fluorescence intensity of markers indicated in A. * $p<0.05$, *** $p<$ 0.001 , Mann-Whitney test. C Acetylated- $\alpha$-tubulin/ $\alpha$-tubulin ratio in the AIS or proximal dendrites in control or $15 \mu \mathrm{M}$

SMIFH2-treated neurons. D KIF5C distribution in control or $15 \mu \mathrm{M}$ SMIFH2-treated 5 DIV neurons for $3 \mathrm{~h}$. Insets show magnifications of KIF5C expression in soma. Scale bar $=100 \mu \mathrm{m}$. E KIF5C normalized fluorescence intensity in soma (circles) and axon (squares) in DMSO (black) or SMIFH2 (red) treated neurons. * $p<0.05, * * * p<0.001$, Mann-Whitney test. F KIF5C normalized fluorescence intensity in proximal axon of 5 DIV neurons treated with $15 \mu \mathrm{M}$ SMIFH2 or DMSO. $* p<0.05$, MannWhitney test. Data were acquired from three independent experiments and represented as the mean \pm SEM
A
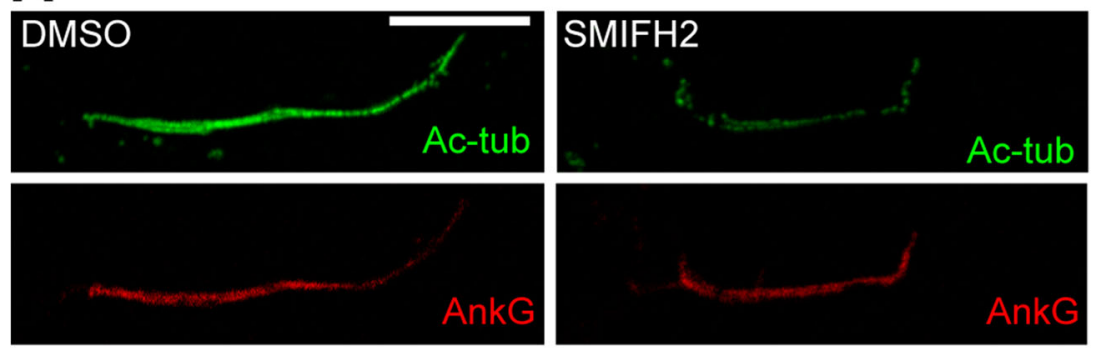

$\beta I V$-spectrin

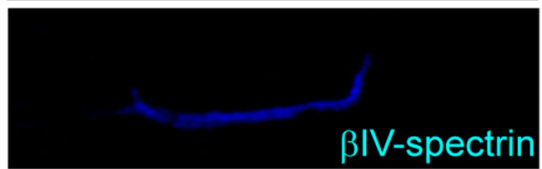

B
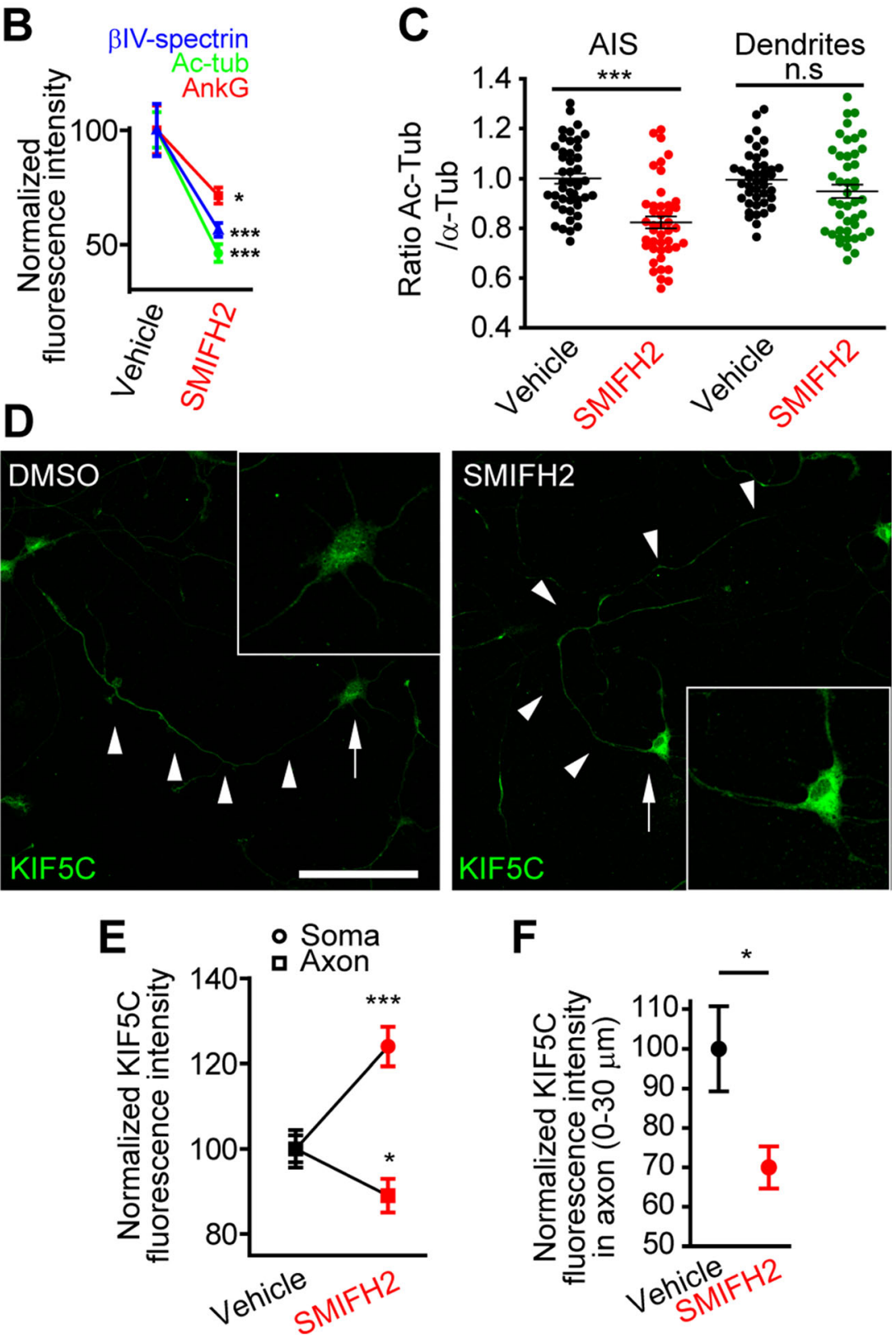

F

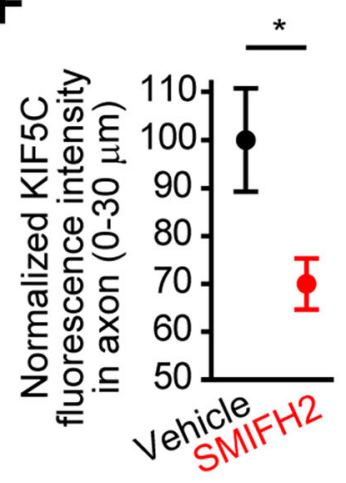


axon length at 3 DIV or dendrites length at 7DIV (Fig. S5G, $\mathrm{H})$. Next, we nucleofected hippocampal neurons with shmDia1-1, shmDia1-2, or srcambled shRNA plasmids for 10 DIV and analyzed mDial staining in the soma of hippocampal neurons, where it is more densely localized (Fig. S5EF). We found that mDial signal was reduced around $45 \%$ (shmDia1-1) and 30\% (shmDia1-2) compared to scrambled shRNA-nucleofected neurons (Fig. S5F). Therefore, we analyzed ankyrinG expression at the AIS of 10 DIV neurons nucleofected with scrambled shRNA or mDial shRNAs (Fig. 5A, B). Both shmDial-1 and shmDia1-2 shRNAs reduced significantly ankyrinG expression (Fig. 5B) to $68 \pm$ $2 \%$ or $78 \pm 2 \%$, respectively, compared to scrambled shRNA $(100 \pm 2 \%)$. The higher reduction produced by shmDial-1 compared to that by shmDial-2 was in agreement with the previously observed higher mDial signal decrease (Fig. S5F). The AnkyrinG intensity profile along the AIS showed that this decline was homogenous all along the AIS (Fig. 5C). Moreover, 10 DIV shmDia1-1-nucleofected neurons showed a significant fluorescence intensity decrease of voltage-gated sodium channels and $\beta I V$-spectrin signals ( $47 \pm$ $6 \%$ and $33 \pm 4 \%$, respectively, Fig. 5D). Besides that, ankyrinG intensity in shmDia1-1-nucleofected neurons was not further reduced after SMIFH2 $(15 \mu \mathrm{M})$ treatment for $3 \mathrm{~h}$ (Fig. 5E, F) compared to that after DMSO treatment $(67 \pm 3 \%$ vs $73 \pm 3 \%$, respectively). However, SMIFH2 produced a significant ankyring reduction in scrambled shRNA-nucleofected neurons $(68 \pm 2 \%$ vs $100 \pm 3 \%$ in DMSO-treated scrambled shRNA neurons), suggesting a SMIFH2 action through mDial inhibition. Next, we investigated whether mDial suppression in later developmental stages did also reproduce the ankyrinG reduction observed after SMIFH2 treatment. Neurons were transfected at 10 DIV with shmDia1-1 or scrambled shRNAs (Fig. 5G) and analyzed 2 days after. We found a significant ankyrinG reduction $(72 \pm 4 \%)$ in neurons transfected with shmDial-1 plasmid compared to scrambled shRNA-transfected neurons (Fig. 5H). These data suggest that neuronal expression of mDial contributes to the maintenance of AIS proteins from early developmental to mature stages of AIS.

\section{AIS Maintenance by Formins Involves Microtubule-Related Mechanisms}

Since our results demonstrate that formin inhibition decreases tubulin acetylation on AIS microtubules, we hypothesized that ankyrinG decrease could be prevented increasing microtubule stability/acetylation. The microtubule end-binding protein, EB1, binds to AIS microtubules [3], interacts with $\mathrm{mDia} 1$ and other mDia proteins, and contributes to stabilize microtubules [57, 58]. Moreover, EB1 allows ankyrinG association to microtubules plus ends [13]. Thus, we expressed EB1-GFP protein in 10 DIV hippocampal neurons before SMIFH2 treatment at 12 DIV (Fig. 6A, B, C). AnkyrinG fluorescence intensity decrease observed in GFP control neurons after SMIFH2 treatment $(65 \% \pm 4 \%$, Fig. $6 \mathrm{~B}, \mathrm{C})$ was significantly prevented in EB1-GFP transfected neurons treated with SMIFH2 $(90 \% \pm 4 \% ; p<0.0001, t$-test, Mann-Whitney test) and happened all along the AIS (Fig. 6C). We did not detect any significant change between GFP:DMSO $(100 \pm 4 \%)$, EB1-GFP:DMSO (99 $\pm 4 \%$ ), and EB1-GFP:SMIFH2 neurons ( $p=0.07$, Kruskal-Wallis test). Then, we tested whether increasing microtubule acetylation maintains ankyrinG levels after formin inhibition or reduction of mDial expression. For that purpose, we inhibited the tubulin deacetylase HDAC6 using Tubastatin A $(10 \mu \mathrm{M})$. The ankyrinG reduction $(84 \pm 2 \%)$ produced by SMIFH2 after $3 \mathrm{~h}$ in 14 DIV neurons was prevented by the HDAC6 inhibitor co-treatment (106 \pm $3 \%$ ) compared to $100 \pm 2 \%$ in control neurons (Fig. 6D). HDAC6 inhibition alone also reduced ankyrinG intensity, as previously described [21]. However, Tubastatin A did not recover the actin-related cisternal organelle loss due to SMIFH2 inhibition (Fig. 6E), in opposition to cisternal organelle recovery in jasplanikinolide-SMIFH2 co-treated neurons (Fig. $3 \mathrm{H}$ ), suggesting a formin-microtubule role on ankyrinG maintenance. We next analyzed whether HDAC6 interference RNA (shHDAC6) could impair ankyrinG reduction in neurons expressing mDial interference RNA (Fig. 6F, G). As happened after HDAC6 inhibition, shHDAC6 reduced ankyrinG intensity by $20 \%$ due to higher general tubulin acetylation, as previously described [21,22]. This reduction was higher all along the AIS in neuron-expressing shmDia1-1 (62 $\pm 4 \%$, Fig. $6 \mathrm{~F}, \mathrm{G})$. However, neurons co-expressing shmDia1-1 and shHDAC6 interference RNAs showed no ankyrinG change $(98 \pm 6 \%)$ compared to $100 \pm 3 \%$ in control scrambled shRNA neurons (Fig. 6F), suggesting a potential compensation of tubulin acetylation that tends to maintain control levels as represented in Figure 6H.

\section{Formin Inhibition or mDia1 Suppression Shortens the AIS}

Next, we analyzed how formin inhibition or mDial downregulation may produce changes on AIS length or position. We first analyzed AIS length in brain slices treated with SMIFH2 $(30 \mu \mathrm{M})$ for $3 \mathrm{~h}$ (Fig. 7A, B). We found that AIS length was significantly different between cortical layers in P30 female mice $(19.39 \pm 0.41 \mu \mathrm{m}$ in layers II/III, $21.49 \pm 0.47 \mu \mathrm{m}$ in layer IV and $23.04 \pm 0.28 \mu \mathrm{m}$ in layers V/VI) and analyzed this parameter by layer groups after SMIFH2 treatment (Fig. 7B). Formin inhibition produced a similar and significant AIS length reduction, around $12 \%$, in each layer group (Fig. 7B). SMIFH2 $(15 \mu \mathrm{M})$ treatment in 10 DIV hippocampal neurons for $3 \mathrm{~h}$ reduced $20 \%$ of the AIS length $(21.15 \pm 0.72 \mu \mathrm{m}$ compared to $27.86 \pm 0.79 \mu \mathrm{m}$ in DMSO-treated neurons, Fig. 7C, D, E). Despite that actin stabilization did not recover 

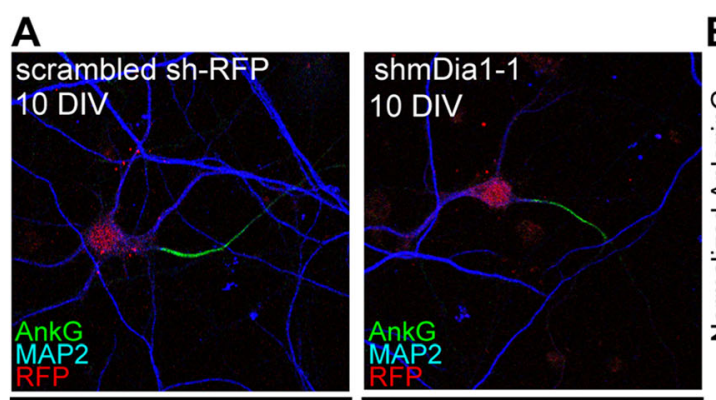

B

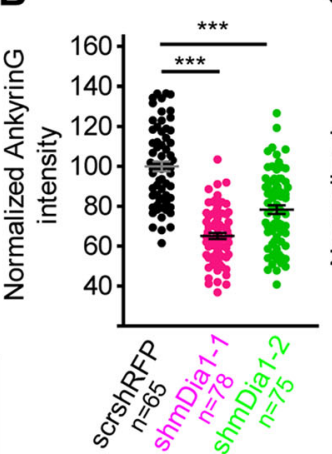

C

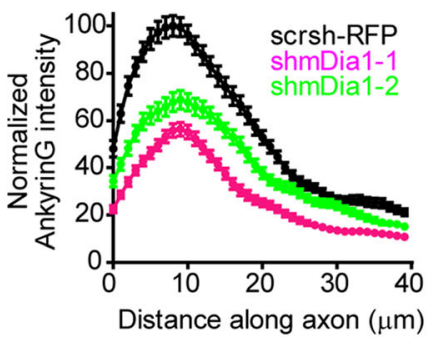

D

ascrsh-RFP shmDia1-1

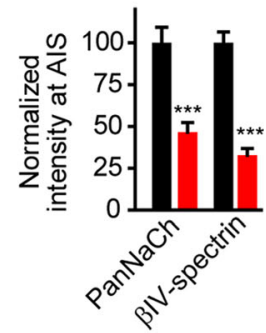

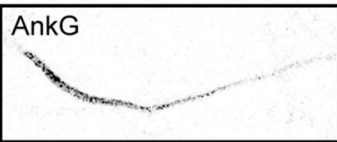

E
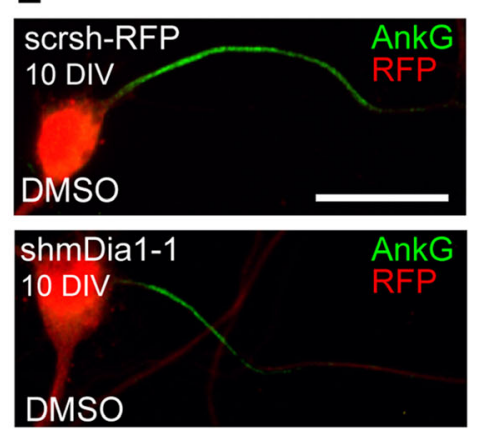

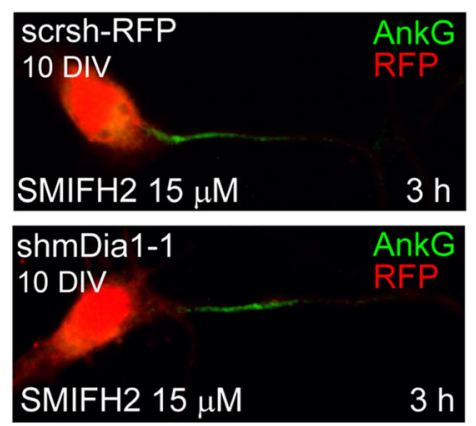

\section{G}
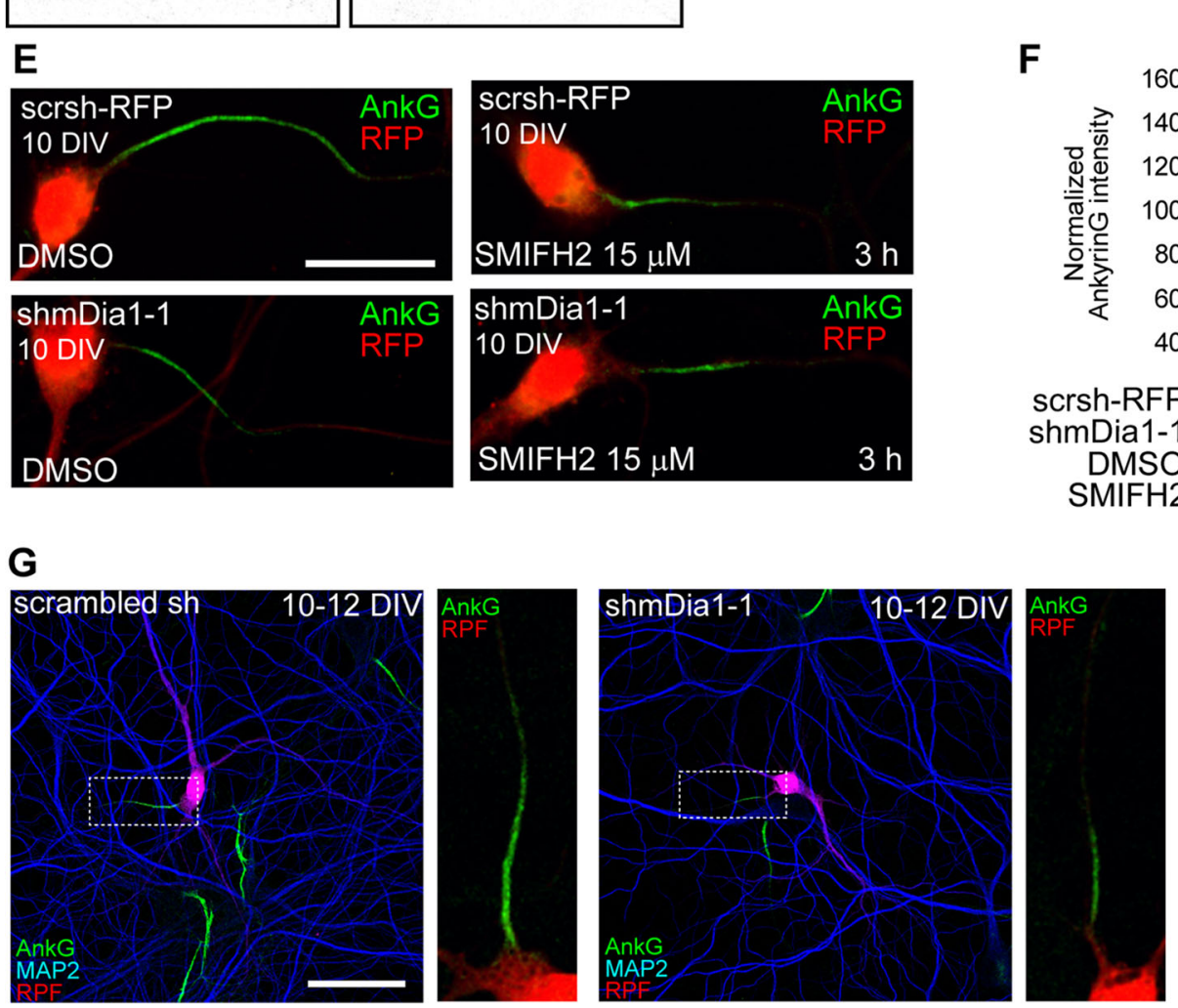

Fig. 5 Suppression of mDial by interference shRNAs decreases AIS proteins accumulation. A Representative images of 10 DIV hippocampal neurons nucleofected with scrambled interference RNA (scrRFP) or mDial interference RNAs (shmDia1-1, shmDia1-2). RFP fluorescence identifies nucleofected neurons (red) and ankyrinG (green) the AIS. Bottom panels show AIS magnifications. Scale bar $=20 \mu \mathrm{m}$. B Normalized ankyrinG fluorescence intensity in the AIS of 10 DIV scrshRFP, shmDia1-1, or shmDia1-2 nucleofected hippocampal neurons. $* * * p<0.001$, one-way analysis of variance, Tukey's multiple comparison test. C Normalized ankyrinG intensity profile along the AIS in 10 DIV-nucleofected neurons shown in B. D Normalized fluorescence intensity of voltage-gated sodium channels ( $\mathrm{PanNaCh}$ ) and $\beta \mathrm{IV}$-spectrin in the AIS of 7 DIV-nucleofected hippocampal neurons. $* * * p<0.001$,

ankyrinG levels, pre-incubation with jasplakinolide $1 \mathrm{~h}$ before SMIFH2 treatment partially prevented AIS shortening (Fig. 7F). We observed the same partial AIS shortening prevention in neurons expressing EB1-GFP after SMIFH2 treatment (Fig. 7G). Transfection of shmDia1-1 plasmid (Fig. 7H, I, J) in 10 DIV hippocampal neurons did also reduce a $20 \%$ the AIS
Mann-Whitney test ( $n=50$ neurons). E AnkyrinG staining in 10 DIV scrsh-RFP- or shmDia1-1-nucleofected hippocampal neurons treated for $3 \mathrm{~h}$ with DMSO or $15 \mu \mathrm{M}$ SMIFH2. Scale bar $=20 \mu \mathrm{m}$. F Normalized ankyrinG fluorescence intensity in the AIS of 10 DIV hippocampal neurons shown in E. n.s., not significant, $* * * p<0.001$, one-way analysis of variance, Tukey's multiple comparison test. G 12 DIV hippocampal neurons lipofected with scrsh-RFP or shmDia1-1 plasmids at 10 DIV. Right panels show AIS magnifications and ankyrinG signal in transfected neurons. Scale bar $=50 \mu \mathrm{m}$. $\mathbf{H}$ Normalized ankyrinG fluorescence intensity $48 \mathrm{~h}$ after transfection of neurons shown in $\mathbf{G}$, $* * * p<0.0001$, unpaired $t$ test $(n=60)$. Data in graphs were acquired from three independent experiments and represented as the mean \pm SEM

length after 2 days $(20.21 \pm 0.72 \mu \mathrm{m}$ vs $25.43 \pm 0.63 \mu \mathrm{m}$ in scrambled shRNA neurons). A similar AIS length reduction $(15 \%)$ happened in shmDia1-1-nucleofected neurons (Fig. $7 \mathrm{~K}, \mathrm{~L})$ after $10 \mathrm{DIV}(21.09 \pm 0.78 \mu \mathrm{m}$ vs $23.85 \pm 0.77 \mu \mathrm{m}$ in scrambled shRNA-nucleofected neurons). AIS starting position or the maximum intensity position did not change in 
A
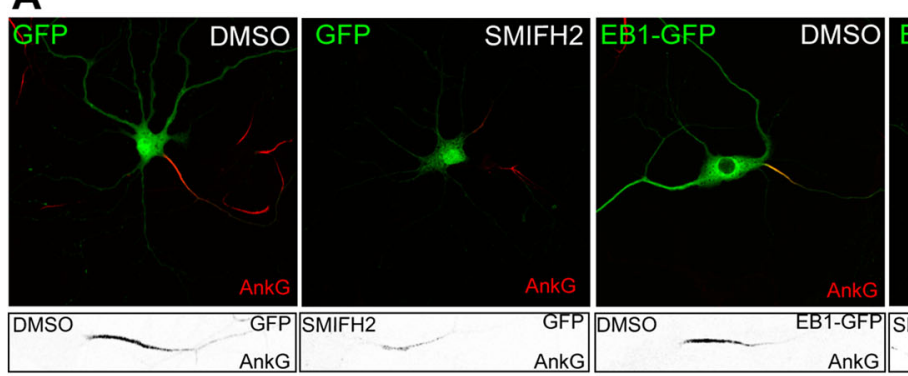

D
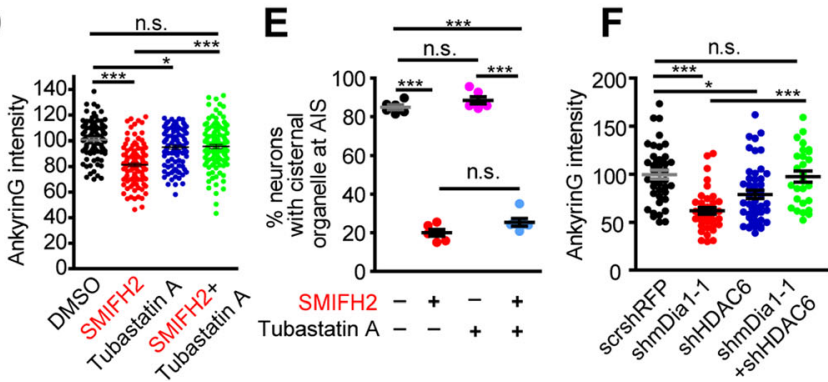

Fig. 6 Increased microtubule acetylation and stabilization impairs formin inhibition-mediated ankyrinG loss. A Representative images of 11 DIV hippocampal neurons transfected with GFP or EB1-GFP for $48 \mathrm{~h}$ and then exposed to DMSO or $15 \mu \mathrm{M}$ SMIFH2 for $3 \mathrm{~h}$. AIS and ankyrinG magnifications are shown in bottom panels. B Normalized ankyrinG fluorescence intensity of neurons shown in A. n.s., not significant, ${ }^{* *} p$ $<0.01, * * * p<0.001$, Kruskal-Wallis, Dunn's multiple comparison test. C AnkyrinG profile along the proximal axon of neurons shown in B. D Normalized ankyrinG intensity in 14 DIV neurons treated with DMSO or $15 \mu \mathrm{M}$ SMIFH2 alone or in combination with the tubulin deacetylase HDAC6 inhibitor Tubastatin A for $3 \mathrm{~h}$. E Percentage of neurons with detectable cisternal organelle after Tubastatin A, SMIFH2, or their combination. Data represent the mean \pm SEM obtained from 6 independent

SMIFH2-treated neurons or neuron-transfected or nucleofected with shmDia1-1 plasmid (Fig. 7E, J, L) proving a distal shortening of the AIS. Finally, we analyzed whether shHDAC6 expression, shown to recover ankyrinG density (Fig. 6F), could prevent mDial shRNA-mediated AIS shortening (Fig. 7M). AISs were again shorter in shmDia1-1-transfected neurons $(28.25 \pm 1.71 \mu \mathrm{m}$ vs $34.41 \pm$ $1.40 \mu \mathrm{m}$ in scrambled shRNA neurons), while those expressing shHDAC6 and shmDia1-1 interference RNAs had AISs not significantly shorter $(32.69 \pm 1.53 \mu \mathrm{m})$. Neurons only expressing shHDAC6 had slightly but significantly shorter AISs $(31.19 \pm 1.05 \mu \mathrm{m})$. From our results, we propose a dual role of formins in AIS actin and microtubules, where microtubule stability/acetylation contributes to maintain mainly ankyrinG density and AIS length, while actin modulation is only involved in AIS length regulation (Fig. 7N).

\section{Discussion}

Axon initial segment plays an important role in the control of intrinsic neuronal excitability, maintains neuronal polarity,
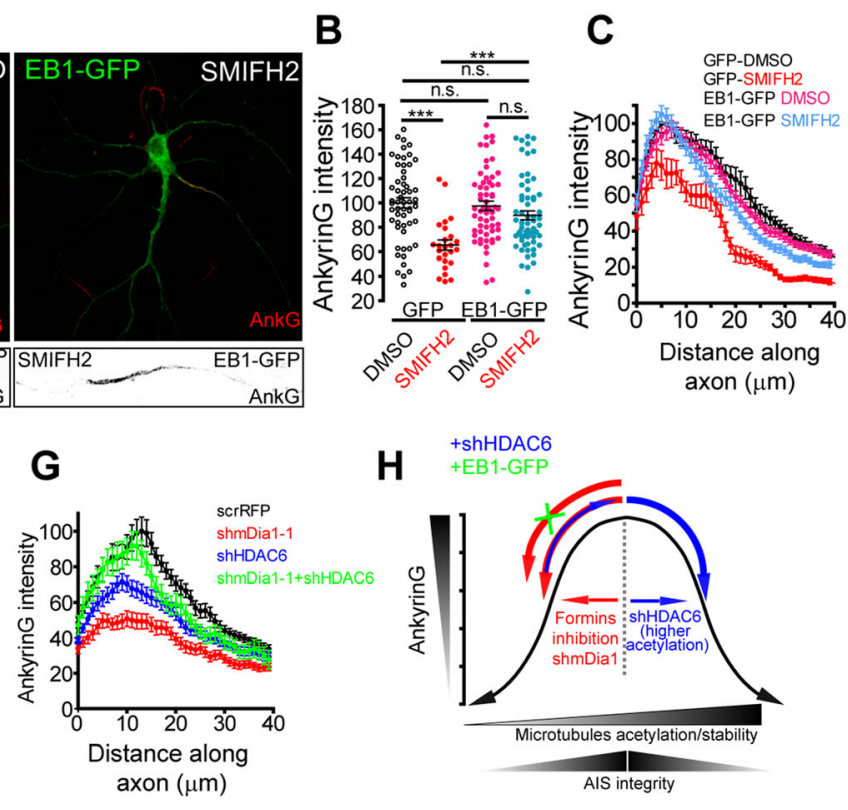

experiments. F Normalized ankyrinG intensity in 7 DIV hippocampal neurons nucleofected with shmDia1-1, shHDAC6, or their combination. Scrsh-RFP nucleofected neurons were used as control to normalize data. n.s., not significant, $* p<0.05, * * * p<0.001$, Kruskal-Wallis, Dunn's multiple comparison test. G AnkyrinG profile along the proximal axon of neurons shown in $\mathbf{F}$. Data were acquired from three independent experiments and represented as the mean \pm SEM. H Schematic representation summarizing the role of formins in the regulation of ankyrinG levels. Decreased formin activity leads to lower acetylation and stability that is counteracted by increasing microtubule acetylation through shHDAC6 or stability by EB1. High tubulin acetylation by shHDAC6 alone also decreases ankyrinG levels

and is capable of a high and dynamic structural plasticity [9, 59]. These important roles require not fully understood mechanisms that modulate AIS cytoskeleton. In our study, we hypothesized that formins, as important cytoskeleton regulators $[34,39]$ that participate in different cellular mechanisms and cell polarity [60], may regulate AIS cytoskeleton contributing to its maintenance and plasticity.

We report that formin activity is necessary to maintain structural ankyrinG and $\beta I V$-spectrin protein or sodium channel density at the AIS. A short time inhibition of formins, using $15 \mu \mathrm{M}$ SMIFH2 concentration lower than the $\mathrm{IC}_{50}(28$ $\mu \mathrm{M})$ previously described in $3 \mathrm{~T} 3$ fibroblasts [51], is enough to decrease ankyrinG density, both in vitro and ex vivo. We also detected a myosin II light chain phosphorylation (pMLC) decrease, previously identified in relation to AIS plasticity [14], and a recent study proposed a SMIFH2 effect on myosins [61] in filopodia adhesion. However, even high SMIFH2 concentrations up to $100 \mu \mathrm{M}$ do not affect non-muscle myosin II phosphorylation [62], suggesting a decrease due to AIS structural protein modifications. In fact, although SMIFH2 (30 $\mu \mathrm{M})$ effects are reversible upon washout on fibroblasts in a short time [51], we found that SMIFH2-induced modifications 

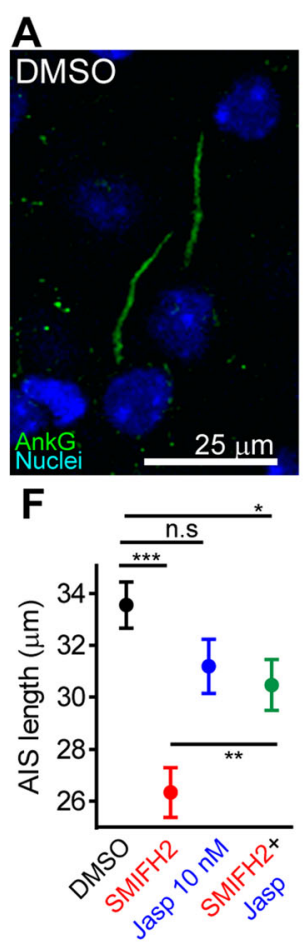

K

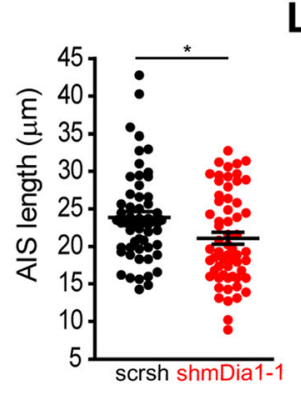

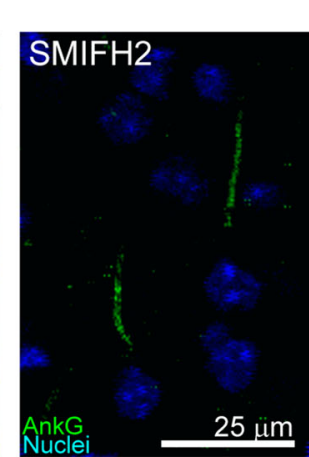

G

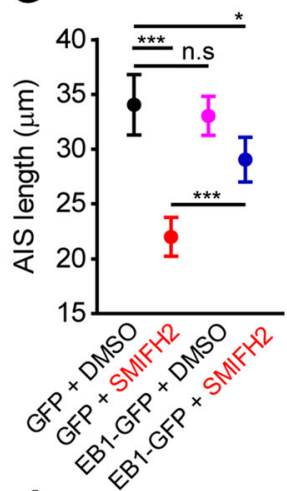

$\mathbf{L}$

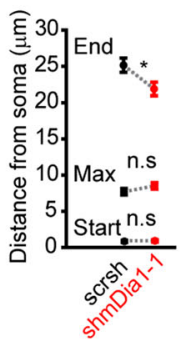

B

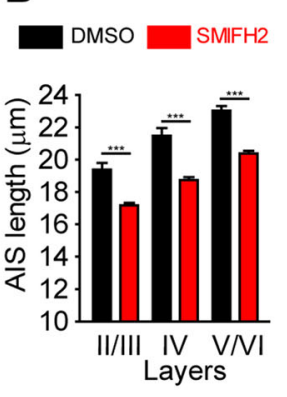

$\mathbf{H}$
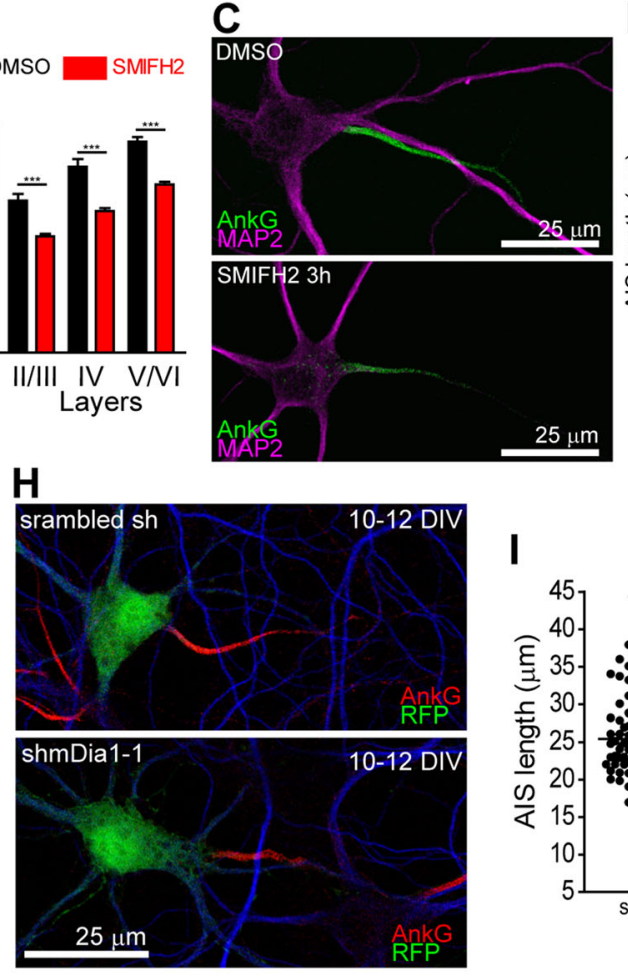

D

E
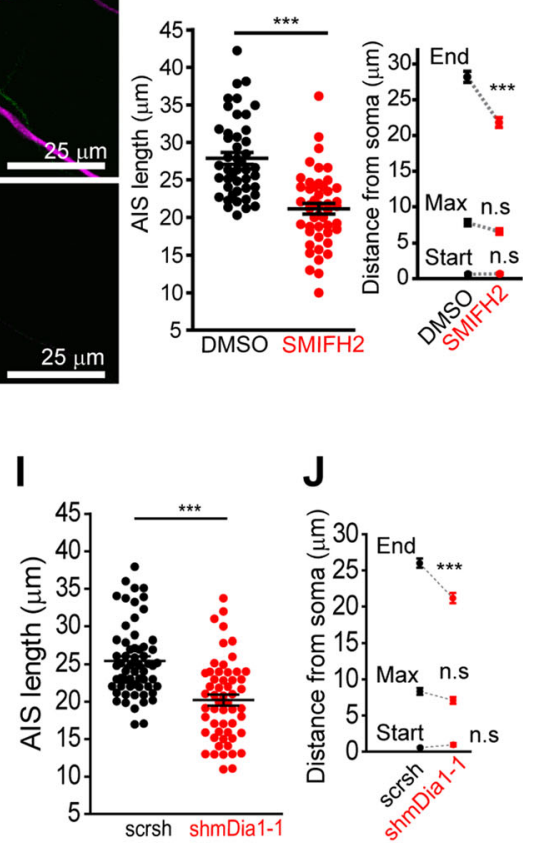

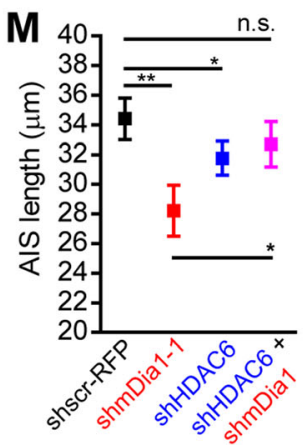

N

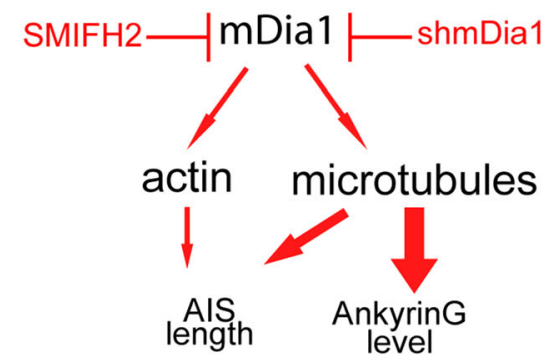

Fig. 7 Decreased formin activity leads to AIS shortening prevented by actin and microtubule stabilization. A Representative images of AISs (green) in layer IV of P30 mice cortical sections treated with DMSO or $30 \mu \mathrm{M}$ SMIFH2. Nuclei are stained with bis-benzimide (blue). Scale bar $=25 \mu \mathrm{m}$. B Quantification of AIS length, in 150 neurons from each layer in 3 independent experiments, in the presence (red) or absence (black) of $30 \mu \mathrm{M}$ SMIFH2 in layers II/III, IV, or V/VI. *** $p<0.0001$, MannWhitney test. C Representative images of AISs in 10 DIV neurons treated with DMSO or SMIFH2 $15 \mu \mathrm{M}$ for $3 \mathrm{~h}$. D Quantification of AIS length in DMSO- or SMIFH2-treated neurons shown in C (60 neurons from 3 experiments). E Mean \pm SEM start, maximum, and end position in DMSO- or SMIFH2-treated neurons. F AIS length in neurons treated with SMIFH2 $(15 \mu \mathrm{M})$ and/or jasplakinolide $(10 \mathrm{nM}), n=80$ neurons from 3 experiments. (G AIS length in GFP- or EB1-GFP-lipofected

neurons treated with SMIFH2 $(15 \mu \mathrm{M})$ or DMSO (60 neurons from 3 experiments). $\mathbf{H}$ Representative images of AISs in 12 DIV shmDia1-1- or scrsh-RFP-transfected neurons for $48 \mathrm{~h}$. I-L Quantification of AIS length in 12 DIV shmDia1-1-transfected neurons (I) and 10 DIV-nucleofected neurons $(\mathbf{K})$. Mean \pm SEM start, maximum, and AIS end position of these neurons are shown in $\mathbf{I}$ and $\mathbf{K}$, respectively. M Quantification of AIS length in 12 DIV neurons transfected with shmDia1-1 and/or shHDAC6 plasmids for $48 \mathrm{~h}$. Data are from 3 independent experiments and 40 neurons by condition. Data are represented as the mean \pm SEM. $n$. s., not significant, $* p<0.05, * * p<0.01, * * * p<0.0001$, Mann-Whitney test. $\mathbf{N}$ Schematic representation that summarizes the role of formins in the regulation of ankyrinG levels and AIS length. Reduced formin activity or mDial actin modifications only affect AIS length, while microtubule modifications reduce AIS length and ankyrinG levels

after $3 \mathrm{~h}$ are not reversible in neurons and led to a later loss of axonal polarity, as previously described after ankyrinG knockdown [1]. Polarity loss happens also in mDia-deficient mice that show a disrupted integrity of their neuroepithelium [42], while in epithelial cells, mDial knockdown do not affect the formation of tight junctions, but their protein content decreases and epithelial barrier is compromised [40] as observed in our study in neurons. The effects produced by SMIFH2 could be associated to different formins expressed in neurons, as it is a general formin inhibitor [51]. However, we show that two different mDial shRNAs also mimic SMIFH2-mediated ankyrinG, $\beta$ IV-spectrin, and sodium 
channel density decrease, maintaining a correlation between mDial staining reduction in soma and ankyrinG decrease. These results are in line with previous results in neurons demonstrating that mDial is the primary target of SMIFH2 [49] and our results showing any further change in ankyrinG density after SMIFH2 treatment in neurons expressing mDia1 interference RNA.

Previous studies identified mDial in dendrites [49], Golgi, or axonal growth cones [44]. mDial antibodies used in this study, including one validated in knockout cells, detected mDial staining at the AIS. However, most mDial antibodies signal and CAmDia1-GFP signal were detected in soma, dendrites, and axons opening the possibility that SMIFH2 effects on AIS composition and structure may come from changes in these structures. Some studies suggest that higher AIS protein density correlates with a more complex dendritic arbor [63, 64]. Previous studies have shown that mDial interference RNAs reduce dendritic ramification in 18 DIV hippocampal neurons [65], while a higher ramification index is observed in 21 DIV cortical neurons after 7 days without affecting spine density [49]. We did not find any significant change in dendrite length after 7 DIV in neurons nucleofected with mDial shRNAs. Thus, ankyrinG decrease found in our study does not seem to be related to changes in dendrites, although we cannot completely discard an effect due to presynaptic or postsynaptic activity changes that may affect AIS structure and composition. Indeed, mDial suppression do not alter spine density or modify mEPSC frequency or amplitude [49] in physiological conditions, but SMIFH2 (30 $\mu \mathrm{M})$-mediated formin inhibition dramatically disrupts synaptic recycling in hippocampal neurons [43], what can modify neuronal inputs and generate changes on AIS structure.

Our data show changes in AIS actin and microtubule cytoskeleton after formin inhibition. Indeed, formins are important regulators of actin nucleation and elongation [66], and regulate microtubule dynamics and stability [57, 67]. We found that formin inhibition was able to disrupt actin filaments and cisternal organelle. Actin cytoskeleton stabilization by jasplakinolide did partially impaired cisternal organelle loss and AIS shortening, but did not impair ankyrinG density decrease. Thus, formin inhibition-mediated actin modifications seem to play only a minor role on AIS length, due to cisternal organelle alterations, previously shown to play a role in AIS length plasticity [27]. Moreover, actin rings do not change during AIS developmental reorganization and shortening [68]. In this sense, mDia1 modulates actin-independent mechanisms, such as the spine density recovery, after $\mathrm{A} \beta$ peptide treatment, by an actin-binding-deficient K994A mDial mutant [67]. We found that formin inhibition decreased tubulin acetylation in AIS microtubules, and not in proximal dendrites, which correlated with ankyrinG decrease. The AIS contains stable microtubules characterized by high detyrosinated and acetylated-tubulin levels compared to dendrites [19, 21], which influences the binding and motility of kinesin-1. Indeed, mDial contributes to the control of microtubule dynamics and increases tubulin acetylation [54], while decreased mDial expression leads to lower detyrosinated tubulin levels [49]. This explains the reduced kinesin-1 localization to the axon and ankyrinG density after formin inhibition. However, ankyrinG density and AIS length remain to control levels when the tubulin deacetylase HDAC6 is inhibited or knockdown in SMIFH2-treated neurons or neurons expressing mDial shRNA. HDAC6 knockdown also decreases AIS length and ankyrinG density, as previously studied [21], to a lesser extent than mDia1 knockdown. A suitable explanation is that both decreased or increased tubulin acetylation modify AIS integrity and lower ankyrinG density. Formin inhibition reduces tubulin acetylation, but HDAC6 suppression increases tubulin acetylation and compensate ankyrinG loss (Fig. 6H). Actually, HDAC6 inhibition compensates transport deficits in Huntington's disease promoting kinesin-1 recruitment by increasing microtubule acetylation [69]. Previous studies showed that HDAC6 and mDia formins collaborate to modulate cytoskeleton [70]. In fact, HDAC6 cooperates with Dial in the formation of links between actin filaments and microtubules, affecting microtubule dynamics [71]. Besides, HDAC6 can physically associate to EB1 [72] that has been proposed to function downstream of mDial in microtubule stabilization [58] and can explain ankyrinG density and AIS length maintenance in neurons over-expressing EB1 when formins are inhibited. Moreover, EB1 not only participates in microtubule dynamics regulation $[58,73]$, but also is a component of the AIS [3], binding ankyrinG to microtubules [74].

Ankyring serves as the anchoring scaffold for voltage-gated sodium channels which density also decreased. In effect, lack of formin activity led to a reduced intrinsic neuronal excitability compatible with a sodium channel density decrease. Despite that the spike number is decreased, spike amplitude was not modified, which can be explained by the higher sensitivity of spike frequency compared to the mild reduction in sodium channel density $(\sim 20 \%$, Fig. $1 \mathrm{E})$ [75] observed after formin inhibition. In addition, formin inhibition depolarized the spike threshold, both in hippocampal and in cortical neurons. Such spike threshold increase may result from the reduction in sodium channel density or AIS length decrease due to the disorganization of the AIS by a formin inhibitor. In fact, a lower spike threshold has been associated to sodium channel density increase during AIS development in nucleus magnocellularis (NM) neurons [68]. Otherwise, spike threshold depolarization happens after AIS shortening due to blast wave exposure without any change in protein density [76]. A recent work suggests a differential regulation of sodium channel accumulation and AIS cytoskeleton reorganization, which work synergistically to optimize neuronal output [68]. 
In conclusion, our study describes a role of formins, and specifically mDial, in the maintenance of AIS protein density and AIS length because of its direct or indirect action on AIS microtubules, but to a lesser extent on AIS actin-related structures (Fig. 7N). Formin loss of function or mutation is a characteristic in several mental disorders, as well as in neurodegenerative diseases [44]. Further studies are necessary to understand which physiological or pathological stimulus can modulate neuronal cytoskeleton through formins or mDial at the AIS or other neuronal compartments in order to regulate AIS structural plasticity.

Supplementary Information The online version contains supplementary material available at https://doi.org/10.1007/s12035-021-02531-6.

Acknowledgements We thank the people of the confocal microscopy facility at Cajal Institute for their help on image acquisition. We thank Dr Wandosell for critical reading of the manuscript.

Code Availability Not applicable

Author Contribution WZ, JJG, PM, MR, DD, and MC performed data analyses; WZ, PM, MC, DR, BA, and NB-G performed experiments. JJG., WZ, and DD contributed to experimental design and data analysis supervision. JJG wrote the paper. WZ, DD, and PM revised the manuscript.

Funding Open Access funding provided thanks to the CRUE-CSIC agreement with Springer Nature. The work was supported by a grant from Ministerio de Ciencia y Universidades (RTI2018-095156-B-100) to JJG and INSERM funding to DD. Wei Zhang was supported by a fellowship from China Scholarship Council (No.201506300085) and Beatriz Achon by a Master fellowship from Universidad Autónoma de Madrid.

Data Availability Data generated and analyzed during this study are included in the published article and its supplementary information files.

\section{Declarations}

Ethics Approval and Consent to Participate Animals, Swiss CD1 mice, were housed in the Experimental Animal facility at Cajal Institute and maintained according to national legislation (53/2013, BOE no. 1337) and guidelines of the Council of the European Communities (2010/63/ UE). All protocols and animal experiments were previously approved by the CSIC bioethics committee and Comunidad de Madrid (PROEX-358/ $15)$.

\section{Consent for Publication Not applicable}

Competing Interests The authors declare no competing interests.

Open Access This article is licensed under a Creative Commons Attribution 4.0 International License, which permits use, sharing, adaptation, distribution and reproduction in any medium or format, as long as you give appropriate credit to the original author(s) and the source, provide a link to the Creative Commons licence, and indicate if changes were made. The images or other third party material in this article are included in the article's Creative Commons licence, unless indicated otherwise in a credit line to the material. If material is not included in the article's Creative Commons licence and your intended use is not permitted by statutory regulation or exceeds the permitted use, you will need to obtain permission directly from the copyright holder. To view a copy of this licence, visit http://creativecommons.org/licenses/by/4.0/.

\section{References}

1. Hedstrom KL, Ogawa Y, Rasband MN (2008) AnkyrinG is required for maintenance of the axon initial segment and neuronal polarity. J Cell Biol 183(4):635-640. https://doi.org/10.1083/jcb.200806112

2. Nakada C, Ritchie K, Oba Y, Nakamura M, Hotta Y, Iino R, Kasai RS, Yamaguchi K, Fujiwara T, Kusumi A (2003) Accumulation of anchored proteins forms membrane diffusion barriers during neuronal polarization. Nat Cell Biol 5(7):626-632. https://doi.org/10. 1038/ncb1009

3. Nakata T, Hirokawa N (2003) Microtubules provide directional cues for polarized axonal transport through interaction with kinesin motor head. J Cell Biol 162(6):1045-1055. https://doi.org/10.1083/ jcb.200302175

4. Sobotzik JM, Sie JM, Politi C, Del Turco D, Bennett V, Deller T, Schultz C (2009) AnkyrinG is required to maintain axo-dendritic polarity in vivo. Proc Natl Acad Sci U S A 106(41):17564-17569. https://doi.org/10.1073/pnas.0909267106

5. Song AH, Wang D, Chen G, Li Y, Luo J, Duan S, Poo MM (2009) A selective filter for cytoplasmic transport at the axon initial segment. Cell 136(6):1148-1160. https://doi.org/10.1016/j.cell.2009.01.016

6. Kole MH, Ilschner SU, Kampa BM, Williams SR, Ruben PC, Stuart GJ (2008) Action potential generation requires a high sodium channel density in the axon initial segment. Nat Neurosci 11(2): 178-186. https://doi.org/10.1038/nn2040

7. Rasband MN (2010) The axon initial segment and the maintenance of neuronal polarity. Nat Rev Neurosci 11(8):552-562. https://doi. org/10.1038/nrn2852

8. Winckler B, Forscher P, Mellman I (1999) A diffusion barrier maintains distribution of membrane proteins in polarized neurons. Nature 397(6721):698-701. https://doi.org/10.1038/17806

9. Grubb MS, Shu Y, Kuba H, Rasband MN, Wimmer VC, Bender KJ (2011) Short- and long-term plasticity at the axon initial segment. J Neurosci 31(45):16049-16055. https://doi.org/10.1523/ JNEUROSCI.4064-11.2011

10. Garrido JJ, Giraud P, Carlier E, Fernandes F, Moussif A, Fache MP, Debanne D, Dargent B (2003) A targeting motif involved in sodium channel clustering at the axonal initial segment. Science 300(5628): 2091-2094. https://doi.org/10.1126/science.1085167

11. Jenkins SM, Bennett V (2001) Ankyrin-G coordinates assembly of the spectrin-based membrane skeleton, voltage-gated sodium channels, and L1 CAMs at Purkinje neuron initial segments. J Cell Biol 155(5):739-746. https://doi.org/10.1083/jcb.200109026

12. Komada M, Soriano P (2002) [Beta]IV-spectrin regulates sodium channel clustering through ankyrin-G at axon initial segments and nodes of Ranvier. J Cell Biol 156(2):337-348. https://doi.org/10. $1083 /$ jcb. 200110003

13. Freal A, Fassier C, Le Bras B, Bullier E, De Gois S, Hazan J, Hoogenraad CC, Couraud F (2016) Cooperative interactions between $480 \mathrm{kDa}$ ankyrin-G and $\mathrm{EB}$ proteins assemble the axon initial segment. J Neurosci 36(16):4421-4433. https://doi.org/10.1523/ JNEUROSCI.3219-15.2016

14. Berger SL, Leo-Macias A, Yuen S, Khatri L, Pfennig S, Zhang Y, Agullo-Pascual E, Caillol G, Zhu MS, Rothenberg E, MelendezVasquez CV, Delmar M, Leterrier C, Salzer JL (2018) Localized myosin II activity regulates assembly and plasticity of the axon 
initial segment. Neuron 97(3):555-570 e556. https://doi.org/10. 1016/j.neuron.2017.12.039

15. Harterink M, Vocking K, Pan X, Soriano Jerez EM, Slenders L, Freal A, Tas RP, van de Wetering WJ, Timmer K, Motshagen J, van Beuningen SFB, Kapitein LC, Geerts WJC, Post JA, Hoogenraad CC (2019) TRIM46 organizes microtubule fasciculation in the axon initial segment. J Neurosci 39(25):4864-4873. https://doi.org/10. 1523/JNEUROSCI.3105-18.2019

16. Jones SL, Korobova F, Svitkina T (2014) Axon initial segment cytoskeleton comprises a multiprotein submembranous coat containing sparse actin filaments. J Cell Biol 205(1):67-81. https:// doi.org/10.1083/jcb.201401045

17. Pan X, Cao Y, Stucchi R, Hooikaas PJ, Portegies S, Will L, Martin M, Akhmanova A, Harterink M, Hoogenraad CC (2019) MAP7D2 localizes to the proximal axon and locally promotes kinesin-1mediated cargo transport into the axon. Cell Rep 26(8):19881999 e1986. https://doi.org/10.1016/j.celrep.2019.01.084

18. Sanchez-Ponce D, Munoz A, Garrido JJ (2011) Casein kinase 2 and microtubules control axon initial segment formation. Mol Cell Neurosci 46(1):222-234. https://doi.org/10.1016/j.mcn.2010.09.005

19. Konishi Y, Setou M (2009) Tubulin tyrosination navigates the kinesin-1 motor domain to axons. Nat Neurosci 12(5):559-567. https://doi.org/10.1038/nn.2314

20. Tapia M, Del Puerto A, Puime A, Sanchez-Ponce D, FronzaroliMolinieres L, Pallas-Bazarra N, Carlier E, Giraud P, Debanne D, Wandosell F, Garrido JJ (2013) GSK3 and beta-catenin determines functional expression of sodium channels at the axon initial segment. Cell Molec Life Sci 70(1):105-120. https://doi.org/10.1007/ s00018-012-1059-5

21. Tapia M, Wandosell F, Garrido JJ (2010) Impaired function of HDAC6 slows down axonal growth and interferes with axon initial segment development. PLoS One 5(9):e12908. https://doi.org/10. 1371/journal.pone.0012908

22. Tsushima H, Emanuele M, Polenghi A, Esposito A, Vassalli M, Barberis A, Difato F, Chieregatti E (2015) HDAC6 and RhoA are novel players in Abeta-driven disruption of neuronal polarity. Nat Commun 6:7781. https://doi.org/10.1038/ncomms8781

23. Sohn PD, Huang CT, Yan R, Fan L, Tracy TE, Camargo CM, Montgomery KM, Arhar T, Mok SA, Freilich R, Baik J, He M, Gong S, Roberson ED, Karch CM, Gestwicki JE, Xu K, Kosik KS, Gan L (2019) Pathogenic tau impairs axon initial segment plasticity and excitability homeostasis. Neuron 104(3):458-470 e455. https:// doi.org/10.1016/j.neuron.2019.08.008

24. Janssen AFJ, Tas RP, van Bergeijk P, Oost R, Hoogenraad CC, Kapitein LC (2017) Myosin-V induces cargo immobilization and clustering at the axon initial segment. Front Cell Neurosci 11:260. https://doi.org/10.3389/fncel.2017.00260

25. Papandreou MJ, Leterrier C (2018) The functional architecture of axonal actin. Mol Cell Neurosci 91:151-159. https://doi.org/10. 1016/j.men.2018.05.003

26. Benedeczky I, Molnar E, Somogyi P (1994) The cisternal organelle as a $\mathrm{Ca}(2+)$-storing compartment associated with GABAergic synapses in the axon initial segment of hippocampal pyramidal neurones. Exp Brain Res 101(2):216-230

27. Schluter A, Del Turco D, Deller T, Gutzmann A, Schultz C, Engelhardt M (2017) Structural plasticity of synaptopodin in the axon initial segment during visual cortex development. Cereb Cortex 27(9):4662-4675. https://doi.org/10.1093/cercor/bhx208

28. Sanchez-Ponce D, Blazquez-Llorca L, DeFelipe J, Garrido JJ, Munoz A (2012) Colocalization of alpha-actinin and synaptopodin in the pyramidal cell axon initial segment. Cereb Cortex 22(7): 1648-1661. https://doi.org/10.1093/cercor/bhr251

29. Dogterom M, Koenderink GH (2019) Actin-microtubule crosstalk in cell biology. Nat Rev Mol Cell Biol 20(1):38-54. https://doi.org/ 10.1038/s41580-018-0067-1
30. Qu Y, Hahn I, Webb SE, Pearce SP, Prokop A (2017) Periodic actin structures in neuronal axons are required to maintain microtubules. Mol Biol Cell 28(2):296-308. https://doi.org/10.1091/mbc.E16-10-0727

31. Balasanyan V, Watanabe K, Dempsey WP, Lewis TL Jr, Trinh LA, Arnold DB (2017) Structure and function of an actin-based filter in the proximal axon. Cell Rep 21(10):2696-2705. https://doi.org/10. 1016/j.celrep.2017.11.046

32. Schonichen A, Geyer M (2010) Fifteen formins for an actin filament: a molecular view on the regulation of human formins. Biochim Biophys Acta 1803(2):152-163. https://doi.org/10.1016/ j.bbamcr.2010.01.014

33. Higgs HN, Peterson KJ (2005) Phylogenetic analysis of the formin homology 2 domain. Mol Biol Cell 16(1):1-13. https://doi.org/10. 1091/mbc.e04-07-0565

34. Bartolini F, Gundersen GG (2010) Formins and microtubules. Biochim Biophys Acta 1803(2):164-173. https://doi.org/10.1016/ j.bbamcr.2009.07.006

35. Kuhn S, Geyer M (2014) Formins as effector proteins of Rho GTPases. Small GTPases 5:e29513. https://doi.org/10.4161/sgtp.29513

36. Ramalingam N, Zhao H, Breitsprecher D, Lappalainen P, Faix J, Schleicher M (2010) Phospholipids regulate localization and activity of mDia1 formin. Eur J Cell Biol 89(10):723-732. https://doi. org/10.1016/j.ejcb.2010.06.001

37. Courtemanche N (2018) Mechanisms of formin-mediated actin assembly and dynamics. Biophys Rev 10(6):1553-1569. https://doi. org/10.1007/s12551-018-0468-6

38. Wallar BJ, Alberts AS (2003) The formins: active scaffolds that remodel the cytoskeleton. Trends Cell Biol 13(8):435-446. https://doi.org/10.1016/s0962-8924(03)00153-3

39. Fernandez-Barrera J, Alonso MA (2018) Coordination of microtubule acetylation and the actin cytoskeleton by formins. Cell Molec Life Sci 75(17):3181-3191. https://doi.org/10.1007/s00018-018-2855-3

40. Acharya BR, Wu SK, Lieu ZZ, Parton RG, Grill SW, Bershadsky AD, Gomez GA, Yap AS (2017) Mammalian diaphanous 1 mediates a pathway for E-cadherin to stabilize epithelial barriers through junctional contractility. Cell Rep 18(12):2854-2867. https://doi. org/10.1016/j.celrep.2017.02.078

41. Herzog D, Loetscher P, van Hengel J, Knusel S, Brakebusch C, Taylor V, Suter U, Relvas JB (2011) The small GTPase RhoA is required to maintain spinal cord neuroepithelium organization and the neural stem cell pool. J Neurosci 31(13):5120-5130. https://doi. org/10.1523/JNEUROSCI.4807-10.2011

42. Thumkeo D, Shinohara R, Watanabe K, Takebayashi H, Toyoda Y, Tohyama K, Ishizaki T, Furuyashiki T, Narumiya S (2011) Deficiency of $\mathrm{mDia}$, an actin nucleator, disrupts integrity of neuroepithelium and causes periventricular dysplasia. PLoS One 6 (9):e25465. https://doi.org/10.1371/journal.pone.0025465

43. Ganguly A, Tang Y, Wang L, Ladt K, Loi J, Dargent B, Leterrier C, Roy S (2015) A dynamic formin-dependent deep F-actin network in axons. J Cell Biol 210(3):401-417. https://doi.org/10.1083/jcb. 201506110

44. Kawabata Galbraith K, Kengaku M (2019) Multiple roles of the actin and microtubule-regulating formins in the developing brain. Neurosci Res 138:59-69. https://doi.org/10.1016/j.neures.2018.09.008

45. Del Puerto A, Fronzaroli-Molinieres L, Perez-Alvarez MJ, Giraud P, Carlier E, Wandosell F, Debanne D, Garrido JJ (2015) ATP$\mathrm{P} 2 \mathrm{X} 7$ receptor modulates axon initial segment composition and function in physiological conditions and brain injury. Cereb Cortex 25(8):2282-2294. https://doi.org/10.1093/cercor/bhu035

46. Kaech S, Banker G (2006) Culturing hippocampal neurons. Nat Protoc 1(5):2406-2415. https://doi.org/10.1038/nprot.2006.356

47. Debanne D, Boudkkazi S, Campanac E, Cudmore RH, Giraud P, Fronzaroli-Molinieres L, Carlier E, Caillard O (2008) Pairedrecordings from synaptically coupled cortical and hippocampal neurons in acute and cultured brain slices. Nat Protoc 3(10):1559 1568. https://doi.org/10.1038/nprot.2008.147 
48. Gasselin C, Inglebert Y, Debanne D (2015) Homeostatic regulation of h-conductance controls intrinsic excitability and stabilizes the threshold for synaptic modification in CA1 neurons. J Physiol 593(22):4855-4869. https://doi.org/10.1113/JP271369

49. Qu X, Yuan FN, Corona C, Pasini S, Pero ME, Gundersen GG, Shelanski ML, Bartolini F (2017) Stabilization of dynamic microtubules by mDial drives Tau-dependent Abeta1-42 synaptotoxicity. J Cell Biol 216(10):3161-3178. https://doi.org/ 10.1083/jcb.201701045

50. Grubb MS, Burrone J (2010) Activity-dependent relocation of the axon initial segment fine-tunes neuronal excitability. Nature 465 (7301):1070-1074. https://doi.org/10.1038/nature09160

51. Rizvi SA, Neidt EM, Cui J, Feiger Z, Skau CT, Gardel ML, Kozmin SA, Kovar DR (2009) Identification and characterization of a small molecule inhibitor of formin-mediated actin assembly. Chem Biol 16(11):1158-1168. https://doi.org/10.1016/j.chembiol. 2009.10.006

52. Schafer DP, Jha S, Liu F, Akella T, McCullough LD, Rasband MN (2009) Disruption of the axon initial segment cytoskeleton is a new mechanism for neuronal injury. J Neurosci 29(42):13242-13254. https://doi.org/10.1523/JNEUROSCI.3376-09.2009

53. Bas Orth C, Schultz C, Muller CM, Frotscher M, Deller T (2007) Loss of the cisternal organelle in the axon initial segment of cortical neurons in synaptopodin-deficient mice. J Comp Neurol 504(5): 441-449. https://doi.org/10.1002/cne.21445

54. Thurston SF, Kulacz WA, Shaikh S, Lee JM, Copeland JW (2012) The ability to induce microtubule acetylation is a general feature of formin proteins. PLoS One 7(10):e48041. https://doi.org/10.1371/ journal.pone. 0048041

55. Tas RP, Chazeau A, Cloin BMC, Lambers MLA, Hoogenraad CC, Kapitein LC (2017) Differentiation between oppositely oriented microtubules controls polarized neuronal transport. Neuron 96(6): 1264-1271 e1265. https://doi.org/10.1016/j.neuron.2017.11.018

56. Arakawa Y, Bito H, Furuyashiki T, Tsuji T, Takemoto-Kimura S, Kimura K, Nozaki K, Hashimoto N, Narumiya S (2003) Control of axon elongation via an SDF-1alpha/Rho/mDia pathway in cultured cerebellar granule neurons. J Cell Biol 161(2):381-391. https://doi. org $/ 10.1083 /$ jcb.200210149

57. Bartolini F, Ramalingam N, Gundersen GG (2012) Actin-capping protein promotes microtubule stability by antagonizing the actin activity of mDia1. Mol Biol Cell 23(20):4032-4040. https://doi. org/10.1091/mbc.E12-05-0338

58. Wen Y, Eng CH, Schmoranzer J, Cabrera-Poch N, Morris EJ, Chen M, Wallar BJ, Alberts AS, Gundersen GG (2004) EB1 and APC bind to $\mathrm{mDia}$ to stabilize microtubules downstream of Rho and promote cell migration. Nat Cell Biol 6(9):820-830. https://doi. org/10.1038/ncb1160

59. Bender KJ, Trussell LO (2012) The physiology of the axon initial segment. Annu Rev Neurosci 35:249-265. https://doi.org/10.1146/ annurev-neuro-062111-150339

60. Carramusa L, Ballestrem C, Zilberman Y, Bershadsky AD (2007) Mammalian diaphanous-related formin Dia1 controls the organization of E-cadherin-mediated cell-cell junctions. J Cell Sci $120(\mathrm{Pt}$ 21):3870-3882. https://doi.org/10.1242/jcs.014365

61. Alieva NO, Efremov AK, Hu S, Oh D, Chen Z, Natarajan M, Ong HT, Jegou A, Romet-Lemonne G, Groves JT, Sheetz MP, Yan J, Bershadsky AD (2019) Myosin IIA and formin dependent mechanosensitivity of filopodia adhesion. Nat Commun 10(1): 3593. https://doi.org/10.1038/s41467-019-10964-w

62. Nishimura Y, Shi S, Zhang F, Liu R, Takagi Y, Bershadsky AD, Viasnoff V, Sellers JR (2021) The formin inhibitor, SMIFH2, inhibits members of the myosin superfamily. J Cell Sci 134. https:// doi.org/10.1242/jcs. 253708
63. Gulledge AT, Bravo JJ (2016) Neuron morphology influences axon initial segment plasticity. eNeuro 3(1):ENEURO.0085ENEU15.2016. https://doi.org/10.1523/ENEURO.0085-15.2016

64. Tapia M, Dominguez A, Zhang W, Del Puerto A, Ciorraga M, Benitez MJ, Guaza C, Garrido JJ (2017) Cannabinoid receptors modulate neuronal morphology and ankyrinG density at the axon initial segment. Front Cell Neurosci 11:5. https://doi.org/10.3389/ fncel.2017.00005

65. Hong EH, Kim JY, Kim JH, Lim DS, Kim M, Kim JY (2018) BIG2-ARF1-RhoA-mDial signaling regulates dendritic Golgi polarization in hippocampal neurons. Mol Neurobiol 55(10):77017716. https://doi.org/10.1007/s12035-018-0954-7

66. Goode BL, Eck MJ (2007) Mechanism and function of formins in the control of actin assembly. Annu Rev Biochem 76:593-627. https://doi.org/10.1146/annurev.biochem.75.103004.142647

67. Bartolini F, Moseley JB, Schmoranzer J, Cassimeris L, Goode BL, Gundersen GG (2008) The formin mDia2 stabilizes microtubules independently of its actin nucleation activity. J Cell Biol 181(3): 523-536. https://doi.org/10.1083/jcb.200709029

68. Akter N, Fukaya R, Adachi R, Kawabe H, Kuba H (2020) Structural and functional refinement of the axon initial segment in avian cochlear nucleus during development. J Neurosci 40(35): 6709-6721. https://doi.org/10.1523/JNEUROSCI.3068-19.2020

69. Dompierre JP, Godin JD, Charrin BC, Cordelieres FP, King SJ, Humbert S, Saudou F (2007) Histone deacetylase 6 inhibition compensates for the transport deficit in Huntington's disease by increasing tubulin acetylation. J Neurosci 27(13):3571-3583. https://doi. org/10.1523/JNEUROSCI.0037-07.2007

70. Destaing O, Saltel F, Gilquin B, Chabadel A, Khochbin S, Ory S, Jurdic P (2005) A novel Rho-mDia2-HDAC6 pathway controls podosome patterning through microtubule acetylation in osteoclasts. J Cell Sci 118(Pt 13):2901-2911. https://doi.org/10.1242/ jes. 02425

71. Bershadsky AD, Ballestrem C, Carramusa L, Zilberman Y, Gilquin B, Khochbin S, Alexandrova AY, Verkhovsky AB, Shemesh T, Kozlov MM (2006) Assembly and mechanosensory function of focal adhesions: experiments and models. Eur J Cell Biol 85(3-4): 165-173. https://doi.org/10.1016/j.ejcb.2005.11.001

72. Zilberman Y, Ballestrem C, Carramusa L, Mazitschek R, Khochbin S, Bershadsky A (2009) Regulation of microtubule dynamics by inhibition of the tubulin deacetylase HDAC6. J Cell Sci 122(Pt 19): 3531-3541. https://doi.org/10.1242/jcs.046813

73. Vitre B, Coquelle FM, Heichette C, Garnier C, Chretien D, Arnal I (2008) EB1 regulates microtubule dynamics and tubulin sheet closure in vitro. Nat Cell Biol 10(4):415-421. https://doi.org/10.1038/ ncb1703

74. Leterrier C, Vacher H, Fache MP, d'Ortoli SA, Castets F, AutilloTouati A, Dargent B (2011) End-binding proteins EB3 and EB1 link microtubules to ankyrin $\mathrm{G}$ in the axon initial segment. Proc Natl Acad Sci U S A 108(21):8826-8831. https://doi.org/10.1073/ pnas. 1018671108

75. Madeja M (2000) Do neurons have a reserve of sodium channels for the generation of action potentials? A study on acutely isolated CA1 neurons from the guinea-pig hippocampus. Eur J Neurosci 12 (1):1-7. https://doi.org/10.1046/j.1460-9568.2000.00871.x

76. Baalman KL, Cotton RJ, Rasband SN, Rasband MN (2013) Blast wave exposure impairs memory and decreases axon initial segment length. J Neurotrauma 30(9):741-751. https://doi.org/10.1089/neu. 2012.2478

Publisher's Note Springer Nature remains neutral with regard to jurisdictional claims in published maps and institutional affiliations. 